\title{
The relation between the Baum-Connes Conjecture and the Trace Conjecture
}

\author{
Wolfgang Lück* \\ Fachbereich Mathematik und Informatik \\ Westfälische Wilhelms-Universität Münster \\ Einsteinstr. 62 \\ 48149 Münster \\ Germany
}

March 22, 2001

\begin{abstract}
We prove a version of the $L^{2}$-index Theorem of Atiyah, which uses the universal center-valued trace instead of the standard trace. We construct for $G$-equivariant K-homology an equivariant Chern character, which is an isomorphism and lives over the ring $\mathbb{Z} \subset \Lambda^{G} \subset \mathbb{Q}$ obtained from the integers by inverting the orders of all finite subgroups of $G$. We use these two results to show that the Baum-Connes Conjecture implies the modified Trace Conjecture, which says that the image of the standard trace $K_{0}\left(C_{r}^{*}(G)\right) \rightarrow \mathbb{R}$ takes values in $\Lambda^{G}$. The original Trace Conjecture predicted that its image lies in the additive subgroup of $\mathbb{R}$ generated by the inverses of all the orders of the finite subgroups of $G$, and has been disproved by Roy [13].

Key words: Baum-Connes Conjecture, Trace Conjecture, equivariant Chern character, $L^{2}$-index theorem.

Mathematics subject classification 2000: 19L47, 19K56, 55N91
\end{abstract}

\section{Introduction and statements of results}

Throughout this paper let $G$ be a discrete group. The Baum-Connes Conjecture for $G$ says that the assembly map

$$
\operatorname{asmb}^{G}: K_{0}^{G}(\underline{E} G) \rightarrow K_{0}\left(C_{r}^{*}(G)\right)
$$

from the equivariant $K$-homology of the classifying space for proper $G$-actions $\underline{E} G$ to the topological $K$-theory of the reduced $C^{*}$-algebra $C_{r}^{*}(G)$ is bijective [3, page 8], [5, Conjecture 3.1]. In connection with this conjecture Baum and Connes [3, page 21] also made the sometimes so called Trace Conjecture. It says that the image of the composition

$$
K_{0}\left(C_{r}^{*}(G)\right) \stackrel{i}{\rightarrow} K_{0}(\mathcal{N}(G)) \stackrel{\operatorname{tr}_{\mathcal{N}(G)}}{\longrightarrow} \mathbb{R}
$$

\footnotetext{
*email: lueck@math.uni-muenster.de, wwww: http://www.math.uni-muenster.de/u/lueck, FAX: 492518338370
} 
is the additive subgroup of $\mathbb{Q}$ generated by all numbers $\frac{1}{|H|}$, where $H \subset G$ runs though all finite subgroups of $G$. Here $\mathcal{N}(G)$ is the group von Neumann algebra, $i$ the change of rings homomorphism associated to the canonical inclusion $C_{r}^{*}(G) \rightarrow \mathcal{N}(G)$ and $\operatorname{tr}_{\mathcal{N}(G)}$ is the map induced by the standard von Neumann trace $\operatorname{tr}_{\mathcal{N}(G)}: \mathcal{N}(G) \rightarrow \mathbb{C}$. Roy has construced a counterexample to the Trace Conjecture in this form in [13] based on her article [14]. She constructs a group $\Gamma$, whose finite subgroups are all of order 1 or 3, together with an element in $K_{0}^{G}(\underline{E} G)$, whose image under $\operatorname{tr}_{\mathcal{N}(\Gamma)} \circ i \circ$ asmb is $-\frac{1105}{9}$. The point is that $3 \cdot \frac{1105}{9}$ is not an integer. Notice that Roy's example does not imply that the Baum-Connes Conjecture does not hold for $\Gamma$. Since the group $\Gamma$ contains a torsionfree subgroup of index 9 and the Trace Conjecture for torsionfree groups does follow from the Baum-Connes Conjecture, the Baum-Connes Conjecture predicts that the image of $\operatorname{tr}_{\mathcal{N}(\Gamma)} \circ i: K_{0}\left(C_{r}^{*}(\Gamma)\right) \rightarrow \mathbb{R}$ is contained in $\{r \in \mathbb{R} \mid 9 \cdot r \in \mathbb{Z}\}$. So one could hope that the following version of the Trace Conjecture is still true. Denote by

$$
\Lambda^{G}:=\mathbb{Z}\left[\frac{1}{\mid \mathcal{F} \text { in }(G) \mid}\right]
$$

the ring $\mathbb{Z} \subset \Lambda^{G} \subset \mathbb{Q}$ obtained from $\mathbb{Z}$ by inverting all the orders $|H|$ of finite subgroups of $G$. For Roy's group $\Gamma$ this is $\left\{m \cdot 3^{-n} \mid m, n \in \mathbb{Z}, n \geq 0\right\}$ and obviously contains $-\frac{1105}{9}$.

Conjecture 0.2 (Modified Trace Conjecture for a group $G$ ) The image of the composition

$$
K_{0}\left(C_{r}^{*}(G)\right) \stackrel{i}{\rightarrow} K_{0}(\mathcal{N}(G)) \stackrel{\operatorname{tr}_{\mathcal{N}(G)}}{\longrightarrow} \mathbb{R}
$$

is contained in $\Lambda^{G}$.

The motivation for this paper is to prove

Theorem 0.3 The image of the composition

$$
\Lambda^{G} \otimes_{\mathbb{Z}} K_{0}^{G}(\underline{E} G) \stackrel{\mathrm{id} \otimes \mathrm{asmb}^{G}}{\longrightarrow} \Lambda^{G} \otimes_{\mathbb{Z}} K_{0}\left(C_{r}^{*}(G)\right) \stackrel{i}{\rightarrow} \Lambda^{G} \otimes_{\mathbb{Z}} K_{0}(\mathcal{N}(G)) \stackrel{\operatorname{tr}_{\mathcal{N}(G)}}{\longrightarrow} \mathbb{R}
$$

is $\Lambda^{G}$.

In particular the modified Trace Conjecture 0.2 holds for $G$, if the assembly map $\mathrm{asmb}^{G}$ : $K_{0}^{G}(\underline{E} G) \rightarrow K_{0}\left(C_{r}^{*}(G)\right)$ appearing in the Baum-Connes Conjecture is surjective.

In order to prove Theorem 0.3 (actually a generalization of it in Theorem 0.8), we will prove a slight generalization of Atiyah's $L^{2}$-Index Theorem and construct an equivariant Chern character for equivariant $K$-homology of proper $G$-CW-complexes, which is bijective and defined after applying $\Lambda^{G} \otimes_{\mathbb{Z}}-$

Let $M$ be a closed Riemannian manifold and $D^{*}=\left(D^{*}, d^{*}\right)$ be an elliptic complex of differential operators of order 1 on $M$. Denote by $\operatorname{index}\left(D^{*}\right) \in \mathbb{Z}$ its index. Let $\bar{M} \rightarrow M$ be a $G$-covering. Then one can lift $D^{*}$ to an elliptic $G$-equivariant complex $\bar{D}^{*}$. Using the $\operatorname{trace} \operatorname{tr}_{\mathcal{N}(G)}: \mathcal{N}(G) \rightarrow \mathbb{C}$ Atiyah [1] defines its $L^{2}$-index $\operatorname{index}_{\mathcal{N}(G)}\left(\bar{D}^{*}\right) \in \mathbb{R}$ and shows

$$
\operatorname{index}\left(D^{*}\right)=\operatorname{index}_{\mathcal{N}(G)}\left(\bar{D}^{*}\right) .
$$

The $L^{2}$-index theorem of Atiyah implies that the composition

$$
K_{0}^{G}(E G) \stackrel{\operatorname{asmb}^{G}}{\longrightarrow} K_{0}\left(C_{r}^{*}(G)\right) \stackrel{i}{\rightarrow} K_{0}(\mathcal{N}(G)) \stackrel{\operatorname{tr}_{\mathcal{N}(G)}}{\longrightarrow} \mathbb{R}
$$

agrees with the composition

$$
K_{0}^{G}(E G) \stackrel{\operatorname{ind}_{G \rightarrow\{1\}}}{\longrightarrow} K_{0}(B G) \stackrel{K_{0}(\mathrm{pr})}{\longrightarrow} K_{0}(*) \stackrel{\operatorname{asmb}^{\{1\}}}{\longrightarrow} K_{0}\left(C_{r}^{*}(\{1\})\right) \stackrel{\operatorname{dim}_{\mathbb{C}}}{\longrightarrow} \mathbb{Z} \hookrightarrow \mathbb{R} .
$$


Since for a torsionfree group $G$ the spaces $E G$ and $\underline{E} G$ agree, the Baum-Connes Conjecture for a torsionfree group $G$ does imply that the image of $K_{0}\left(C_{r}^{*}(G)\right) \stackrel{i}{\rightarrow} K_{0}(\mathcal{N}(G)) \stackrel{\operatorname{tr}_{\mathcal{N}(G)}}{\longrightarrow} \mathbb{R}$ is $\mathbb{Z}[3$,

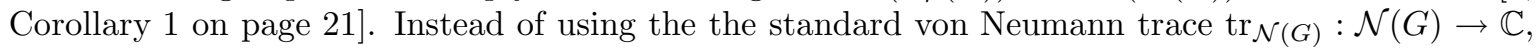

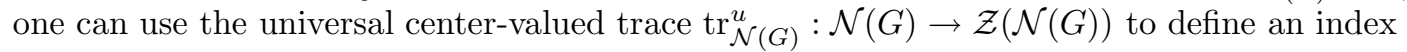

$$
\operatorname{index}_{\mathcal{N}(G)}^{u}\left(\bar{D}^{*}\right) \in \mathcal{Z}(\mathcal{N}(G))
$$

which takes values in the center $\mathcal{Z}(\mathcal{N}(G))$ of the group von Neumann algebra $\mathcal{N}(G)$. Thus we get additional information, namely, for any element $g \in G$, whose conjugacy class $(g)$ is finite, we get a complex number. However, it turns out that the value at classes $(g)$ with $g \neq 1$ is zero and that the value at (1) is the index of $D^{*}$. Namely, we will show in Section 1

Theorem 0.4 Under the conditions above we get in $\mathcal{Z}(\mathcal{N}(G))$

$$
\operatorname{index}_{\mathcal{N}(G)}^{u}\left(\bar{D}^{*}\right)=\operatorname{index}\left(D^{*}\right) \cdot 1_{\mathcal{N}(G)} .
$$

As an illustration we discuss the special case, where $G$ is finite, $M$ is an oriented closed $4 k$ dimensional manifold with free orientation preserving $G$-action and $D^{*}$ is the signature operator. Then Theorem 0.4 reduces to the well-known statement that the equivariant signature

$$
\operatorname{sign}^{G}(M):=\left[H_{2 k}(M)^{+}\right]-\left[H_{2 k}(M)^{-}\right] \in \operatorname{Rep}_{\mathbb{C}}(G)
$$

is equal to $\operatorname{sign}(G \backslash M) \cdot[\mathbb{C} G]$ for $\operatorname{sign}(G \backslash M) \in \mathbb{Z}$ the (ordinary) signature of $G \backslash M$. We mention that this implies $\operatorname{sign}(M)=|G| \cdot \operatorname{sign}(G \backslash M)$. Theorem 0.4 is a special case of Theorem 5.4 but we will need it in the proof of Theorem 5.4 and therefore will have to prove it first.

The second ingredient is a variation of the equivariant Chern character of [11] for equivariant $K$ homology of proper $G-C W$-complexes. Recall that proper means that all isotropy groups are finite. The construction in [11] works for equivariant homology theories with a Mackey structure on the coefficient system in general, but requires to invert all primes. The construction we will give here works after applying $\Lambda^{G} \otimes_{\mathbb{Z}}$ ? and has a different source.

Denote for a proper $G$-CW-complex $X$ by $\mathcal{F}(X)$ the set of all subgroups $H \subset G$, for which $X^{H} \neq \emptyset$, and by

$$
\Lambda^{G}(X):=\mathbb{Z}\left[\frac{1}{\mathcal{F}(X)}\right]
$$

the ring $\mathbb{Z} \subset \Lambda^{G}(X) \subset \Lambda^{G}$ obtained from $\mathbb{Z}$ by inverting the orders of all subgroups $H \in \mathcal{F}(X)$. Denote by

$$
J^{G} \quad \operatorname{resp} . J^{G}(X)
$$

the set of conjugacy classes $(C)$ of finite cyclic subgroups $C \subset G$ resp. the subset $J^{G}(X) \subset J^{G}$ of conjugacy classes $(C)$ of finite cyclic subgroups $C \subset G$, for which $X^{C}$ is non-empty. Obviously $\Lambda^{G}=\Lambda^{G}(\underline{E} G)$ and $J^{G}=J^{G}(\underline{E} G)$ since $\underline{E} G$ is characterized up to $G$-homotopy by the property that $\underline{E} G^{H}$ is contractible (and hence non-empty) for finite $H \subset G$ and empty for infinite $H \subset G$. Let $\bar{C} \subset G$ be a finite cyclic subgroup. Let $C_{G} C$ be the centralizer and $N_{G} C$ be the normalizer of $C \subset G$. Let $W_{G} C$ be the quotient $N_{G} C / C_{G} C$. We will construct an idempotent $\theta_{C} \in \Lambda^{C} \otimes_{\mathbb{Z}} \operatorname{Rep}_{\mathbb{Q}}(C)$ which acts on $\Lambda^{C} \otimes_{\mathbb{Z}} \operatorname{Rep}_{\mathbb{C}}(C)$. We will see in Lemma 3.4 (b) that the cokernel of

$$
\oplus_{D \subset C, D \neq C} \operatorname{ind}_{D}^{C}: \oplus_{D \subset C, D \neq C} \mathbb{Z}\left[\frac{1}{|C|}\right] \otimes_{\mathbb{Z}} \operatorname{Rep}_{\mathbb{C}}(D) \rightarrow \mathbb{Z}\left[\frac{1}{|C|}\right] \otimes_{\mathbb{Z}} \operatorname{Rep}_{\mathbb{C}}(C)
$$


is isomorphic to the image of the idempotent endomorphism

$$
\theta_{C}: \mathbb{Z}\left[\frac{1}{|C|}\right] \otimes_{\mathbb{Z}} \operatorname{Rep}_{\mathbb{C}}(C) \rightarrow \mathbb{Z}\left[\frac{1}{|C|}\right] \otimes_{\mathbb{Z}} \operatorname{Rep}_{\mathbb{C}}(C)
$$

After introducing and proving some preliminary results about modules over a category and representation theory of finite groups in Sections 2 and 3, we will prove in Section 4

Theorem 0.7 Let $X$ be a proper $G$-CW-complex. Put $\Lambda=\Lambda^{G}(X)$ and $J=J^{G}(X)$. Then there is for $p=0,1$ a natural isomorphism called equivariant Chern character

$$
\begin{aligned}
\operatorname{ch}_{p}^{G}(X): \oplus_{(C) \in J} \Lambda \otimes_{\mathbb{Z}} K_{p}\left(C_{G} C \backslash X^{C}\right) \otimes_{\Lambda\left[W_{G} C\right]} \operatorname{im}\left(\theta_{C}: \Lambda \otimes_{\mathbb{Z}} \operatorname{Rep}_{\mathbb{C}}(C) \rightarrow \Lambda \otimes_{\mathbb{Z}} \operatorname{Rep}_{\mathbb{C}}(C)\right) & \cong \underset{\mathbb{Z}}{\cong} \otimes_{\mathbb{Z}} K_{p}^{G}(X) .
\end{aligned}
$$

Notice that the equivariant Chern character of Theorem 0.7 reduces to the obvious isomorphism $K_{0}(G \backslash X) \otimes_{\mathbb{Z}} \operatorname{Rep}_{\mathbb{C}}(\{1\}) \stackrel{\cong}{\longrightarrow} K_{0}^{G}(X)$, if $G$ acts freely on $X$. In the special case, where $G$ is finite, $X$ is the one-point-space $\{*\}$ and $p=0$, the equivariant Chern character reduces to an isomorphism

$$
\begin{aligned}
& \oplus_{(C) \in J^{G}} \mathbb{Z}\left[\frac{1}{|G|}\right] \otimes_{\mathbb{Z}\left[\frac{1}{|G|}\right]\left[W_{G} C\right]} \operatorname{im}\left(\theta_{C}: \mathbb{Z}\left[\frac{1}{|G|}\right] \otimes_{\mathbb{Z}} \operatorname{Rep}_{\mathbb{C}}(C) \rightarrow \mathbb{Z}\left[\frac{1}{|G|}\right] \otimes_{\mathbb{Z}} \operatorname{Rep}_{\mathbb{C}}(C)\right) \\
& \cong \mathbb{Z}\left[\frac{1}{|G|}\right] \otimes_{\mathbb{Z}} \operatorname{Rep}_{\mathbb{C}}(G)
\end{aligned}
$$

This is a strong version of the well-known theorem of Artin that the map induced by induction

$$
\oplus_{(C) \in J^{G}} \mathbb{Q} \otimes_{\mathbb{Z}} \operatorname{Rep}_{\mathbb{C}}(C) \rightarrow \mathbb{Q} \otimes_{\mathbb{Z}} \operatorname{Rep}_{\mathbb{C}}(G)
$$

is surjective for any finite group $G$. Theorem 0.7 gives a computation of $\Lambda^{G} \otimes K_{0}^{G}(\underline{E} G)$, namely

$$
\begin{aligned}
& \oplus_{(C) \in J^{G}} \Lambda^{G} \otimes_{\mathbb{Z}} K_{p}\left(B\left(C_{G} C\right)\right) \otimes_{\Lambda^{G}\left[W_{G} C\right]} \operatorname{im}\left(\theta_{C}: \Lambda^{G} \otimes_{\mathbb{Z}} \operatorname{Rep}_{\mathbb{C}}(C) \rightarrow \Lambda^{G} \otimes_{\mathbb{Z}} \operatorname{Rep}_{\mathbb{C}}(C)\right) \\
& \cong \Lambda^{G} \otimes_{\mathbb{Z}} K_{p}^{G}(\underline{E} G) .
\end{aligned}
$$

Another construction of an equivariant Chern character using completely different methods can be found in [4]. However, it works only after applying $\mathbb{C} \otimes_{\mathbb{Z}}-$ and therefore cannot be used for our purposes here.

In Theorem 5.4 we will identify the composition of the Chern character of Theorem 0.7 with the map

$$
\Lambda^{G} \otimes_{\mathbb{Z}} K_{0}^{G}(\underline{E} G) \stackrel{\mathrm{id} \otimes \mathrm{asmb}^{G}}{\longrightarrow} \Lambda^{G} \otimes_{\mathbb{Z}} K_{0}\left(C_{r}^{*}(G)\right) \stackrel{\mathrm{id} \otimes i}{\longrightarrow} \Lambda^{G} \otimes_{\mathbb{Z}} K_{0}(\mathcal{N}(G))
$$

with an easier to understand and to calculate homomorphism, whose image is obvious from its definition. This will immediately imply

Theorem 0.8 Let $\Lambda^{G}$ resp. $J^{G}$ be the ring resp. set introduced in (0.1) resp. (0.6). Then the image of the composition

$$
\Lambda^{G} \otimes_{\mathbb{Z}} K_{0}^{G}(\underline{E} G) \stackrel{\operatorname{id} \otimes_{\mathbb{Z}} \mathrm{asmb}^{G}}{\longrightarrow} \Lambda^{G} \otimes_{\mathbb{Z}} K_{0}\left(C_{r}^{*}(G)\right) \stackrel{\operatorname{id} \otimes_{\mathbb{Z}} i}{\longrightarrow} \Lambda^{G} \otimes_{\mathbb{Z}} K_{0}(\mathcal{N}(G))
$$

is the image of the map given by induction

$$
\oplus_{(C) \in J^{G}} \text { id } \otimes \operatorname{ind}_{C}^{G}: \oplus_{(C) \in J^{G}} \Lambda^{G} \otimes_{\mathbb{Z}} \operatorname{Rep}_{\mathbb{C}}(C) \rightarrow \Lambda^{G} \otimes_{\mathbb{Z}} K_{0}(\mathcal{N}(G)) .
$$


Now Theorem 0.3 follows from Theorem 0.8 .

The change of rings and K-theory map $l: K_{0}(\mathbb{C} G) \rightarrow K_{0}\left(C_{r}^{*}(G)\right)$ from the algebraic $K_{0}$-group of the complex group ring $\mathbb{C} G$ to the topological $K_{0}$-group of $C_{r}^{*}(G)$ is in general far from being surjective. There is some evidence that it is injective after applying $\Lambda \otimes_{\mathbb{Z}}$ ? (see [11, Theorem 0.1]). Theorem 0.8 gives some evidence for the conjecture that the image of $\Lambda^{G} \otimes_{\mathbb{Z}} K_{0}\left(C_{r}^{*}(G)\right) \stackrel{\operatorname{id} \otimes_{\mathbb{Z} i}}{\longrightarrow} \Lambda^{G} \otimes_{\mathbb{Z}} K_{0}(\mathcal{N}(G))$ agrees with the image of the composition $\Lambda^{G} \otimes_{\mathbb{Z}} K_{0}(\mathbb{C} G) \stackrel{l}{\rightarrow} \Lambda^{G} \otimes_{\mathbb{Z}} K_{0}\left(C_{r}^{*}(G)\right) \stackrel{\text { id } \otimes_{\mathbb{Z}} i}{\longrightarrow} \Lambda^{G} \otimes_{\mathbb{Z}} K_{0}(\mathcal{N}(G))$. The paper is organized as follows

1. The $L^{2}$-index theorem

2. Modules over a category

3. Some representation theory for finite groups

4. The construction of the Chern character

5. The Baum-Connes Conjecture and the Trace Conjecture References

The author wants to thank the Max-Planck-Institute for Mathematics in Bonn for the hospitality during his stay in January and February 2001, when parts of the paper were written.

\section{The $L^{2}$-index theorem}

In this section we prove a slight generalization of the $L^{2}$-index theorem of Atiyah [1]. Let $\bar{M}$ be a Riemannian manifold (without boundary) together with a cocompact free proper action of $G$ by isometries. In other words, $M=G \backslash \bar{M}$ is a closed Riemannian manifold, the projection $p: \bar{M} \rightarrow M$ is a $G$-covering and $\bar{M}$ is equipped with the Riemannian metric induced by the one of $M$. Let $D^{*}=\left(D^{*}, d^{*}\right)$ be an elliptic complex of differential operators $d^{p}: D^{p} \rightarrow D^{p+1}$ of order 1 acting on the space of sections $D^{p}=C^{\infty}\left(E^{p}\right)$ of vector bundles $E^{p} \rightarrow M$. Define $\bar{E}^{p}$ by $p^{*} E^{p}$ and $\bar{D}^{p}$ by $L^{2} C^{\infty}\left(\bar{E}_{p}\right)$. Then $G$-acts on $\bar{E}^{p}$ and $\bar{D}^{p}$. Since differential operators are local operators, there is a unique lift of each operator $d^{p}$ to a $G$-equivariant differential operator $\widehat{d}^{p}: C^{\infty}\left(\bar{E}^{p}\right) \rightarrow C^{\infty}\left(\bar{E}^{p+1}\right)$. We obtain an elliptic $G$-complex $\left(C^{\infty}\left(\bar{E}^{*}\right), \widehat{d}^{*}\right)$. Let $\bar{d}^{p}: \bar{D}^{p} \rightarrow \bar{D}^{p+1}$ be the minimal closure of $\widehat{d}^{p}$ which is the same as its maximal closure [1, Proposition 3.1].

Since $D^{*}$ is elliptic, each cohomology module $H^{p}\left(D^{*}\right):=\operatorname{ker}\left(d^{p}\right) / \operatorname{im}\left(d^{p-1}\right)$ is a finitely generated $\mathbb{C}$-module. Hence we can define the index of the elliptic complex $D^{*}$ by

$$
\operatorname{index}\left(D^{*}\right):=\sum_{p \geq 0} \operatorname{dim}_{\mathbb{C}}\left(H^{p}\left(D^{*}\right)\right) \in \mathbb{Z}
$$

Next we want to define an analogous invariant for the lifted complex $\bar{D}^{*}$. The group von Neumann algebra $\mathcal{N}(G)$ of $G$ is the $*$-algebra $\mathcal{B}\left(l^{2}(G)\right)^{G}$ of all bounded $G$-equivariant operators $l^{2}(G) \rightarrow l^{2}(G)$, where we equip $l^{2}(G)$ with the obvious left $G$-action. Let

$$
\operatorname{tr}_{\mathcal{N}(G)}: \mathcal{N}(G) \rightarrow \mathbb{C}
$$

be the standard von Neumann trace, which sends $f \in \mathcal{N}(G)=\mathcal{B}\left(l^{2}(G)\right)^{G}$ to $\langle f(e), e\rangle_{l^{2}(G)}$, where $e$ denotes the element in $l^{2}(G)$ given by the unit element in $G \subset l^{2}(G)$. Denote by $\mathcal{Z}(\mathcal{N}(G))$ the center of $\mathcal{N}(G)$. There is the universal center-valued trace [7, Theorem 7.1.12 on page 462, Proposition 7.4.5 on page 483 , Theorem 8.2.8 on page 517, Proposition 8.3.10 on page 525, Theorem 8.4.3 on page 532]

$$
\operatorname{tr}_{\mathcal{N}(G)}^{u}: \mathcal{N}(G) \rightarrow \mathcal{Z}(\mathcal{N}(G))
$$


which is uniquely determined by the following two properties:

(a) $\operatorname{tr}^{u}$ is a trace with values in the center, i.e. $\operatorname{tr}^{u}$ is $\mathbb{C}$-linear, for $a \in \mathcal{N}(G)$ with $a \geq 0$ we have $\operatorname{tr}^{u}(a) \geq 0$ and $\operatorname{tr}^{u}(a b)=\operatorname{tr}^{u}(b a)$ for all $a, b \in \mathcal{N}(G)$;

(b) $\operatorname{tr}^{u}(a)=a$ for all $a \in Z(\mathcal{N}(G))$.

The map $\operatorname{tr}^{u}$ has the following further properties:

(c) $\operatorname{tr}^{u}$ is faithful;

(d) $\operatorname{tr}^{u}$ is normal. Equivalently, $\operatorname{tr}^{u}$ is continuous with respect to the ultraweak topology on $\mathcal{N}(G)$;

(e) $\left\|\operatorname{tr}^{u}(a)\right\| \leq\|a\|$ for $a \in \mathcal{N}(G)$

(f) $\operatorname{tr}^{u}(a b)=a \operatorname{tr}^{u}(b)$ for all $a \in Z(\mathcal{N}(G))$ and $b \in \mathcal{N}(G)$;

(g) Let $p$ and $q$ be projections in $\mathcal{N}(G)$. Then $p$ and $q$ are equivalent, i.e. $p=v v^{*}$ and $q=v^{*} v$, if and only if $\operatorname{tr}^{u}(p)=\operatorname{tr}^{u}(q)$;

(h) Any linear functional $f: \mathcal{N}(G) \rightarrow \mathbb{C}$, which is continuous with respect to the norm topology on $\mathcal{N}(G)$ and which is central, i.e. $f(a b)=f(b a)$ for all $a, b \in \mathcal{N}(G)$, factorizes as

$$
\mathcal{N}(G) \stackrel{\operatorname{tr}^{u}}{\longrightarrow} Z(\mathcal{N}(G)) \stackrel{\left.f\right|_{Z(\mathcal{N}(G))}}{\longrightarrow} \mathbb{C}
$$

In particular $\operatorname{tr}_{\mathcal{N}(G)} \circ \operatorname{tr}_{\mathcal{N}(G)}^{u}=\operatorname{tr}_{\mathcal{N}(G)}$.

A Hilbert $\mathcal{N}(G)$-module $V$ is a Hilbert space $V$ together with a $G$-action by isometries such that there exists a Hilbert space $H$ and a $G$-equivariant projection $p: H \otimes l^{2}(G) \rightarrow H \otimes l^{2}(G)$ with the property that $V$ and $\operatorname{im}(p)$ are isometrically $G$-linearly isomorphic. Here $H \otimes l^{2}(G)$ is the tensor product of Hilbert spaces and $G$ acts trivially on $H$ and on $l^{2}(G)$ by the obvious left multiplication. Notice that $p$ is not part of the structure, only its existence is required. We call $V$ finitely generated if $H$ can be choosen to be finite-dimensional.

Our main examples of Hilbert $\mathcal{N}(G)$-modules are the Hilbert spaces $\bar{D}^{p}$ which are isometrically $G$-isomorphic to $L^{2}\left(C^{\infty}\left(E^{p}\right)\right) \otimes l^{2}(G)$. This can be seen using a fundamental domain $\mathcal{F}$ for the $G$ action on $\bar{M}$ which is from a measure theory point of view the same as $M$. A morphism $f: V \rightarrow W$ of Hilbert $\mathcal{N}(G)$-modules is a densely defined closed $G$-equivariant operator. The differentials $\bar{d}^{p}$ are morphisms of Hilbert $\mathcal{N}(G)$-modules.

Let $f: V \rightarrow V$ be a morphism of Hilbert $\mathcal{N}(G)$-modules which is positive. Choose a $G$-projection $p: H \otimes l^{2}(G) \rightarrow H \otimes l^{2}(G)$ and an isometric invertible $G$-equivariant operator $u: \operatorname{im}(p) \rightarrow V$. Let $\left\{b_{i} \mid i \in I\right\}$ be a Hilbert basis for $H$. Let $\bar{f}$ be the composition

$$
H \otimes l^{2}(G) \stackrel{p}{\rightarrow} \operatorname{im}(p) \stackrel{u}{\longrightarrow} V \stackrel{f}{\rightarrow} V \stackrel{u^{-1}}{\longrightarrow} \operatorname{im}(p) \hookrightarrow H \otimes l^{2}(G)
$$

Define the von Neumann trace of $f: V \rightarrow V$ by

$$
\operatorname{tr}_{\mathcal{N}(G)}(f):=\sum_{i \in I}\left\langle\bar{f}\left(b_{i} \otimes e\right), b_{i} \otimes e\right\rangle_{H \otimes l^{2}(G)} \in[0, \infty] .
$$

This is indeed independent of the choice of $p, u$ and the Hilbert basis $\left\{b_{i} \mid i \in I\right\}$. If $V$ is finitely generated, then $\operatorname{tr}_{\mathcal{N}(G)}(f)<\infty$ is always true. Define the von Neumann dimension of a Hilbert $\mathcal{N}(G)$-module $V$ by

$$
\operatorname{dim}_{\mathcal{N}(G)}(V):=\operatorname{tr}_{\mathcal{N}(G)}(\mathrm{id}: V \rightarrow V) \quad \in[0, \infty]
$$


If $V$ is a finitely generated Hilbert $\mathcal{N}(G)$-module, we define the universal center-valued von Neumann dimension

$$
\operatorname{dim}_{\mathcal{N}(G)}^{u}(V):=\operatorname{tr}_{\mathcal{N}(G)}^{u}(\text { id }: V \rightarrow V) \in \mathcal{Z}(\mathcal{N}(G))
$$

analogously to $\operatorname{dim}_{\mathcal{N}(G)}(V)$ replacing $\operatorname{tr}_{\mathcal{N}(G)}$ by $\operatorname{tr}_{\mathcal{N}(G)}^{u}$. Given a finitely generated Hilbert $\mathcal{N}(G)$ module $V$, we have $\operatorname{tr}_{\mathcal{N}(G)}\left(\operatorname{dim}_{\mathcal{N}(G)}^{u}(V)\right)=\operatorname{dim}_{\mathcal{N}(G)}(V)$.

Define the $L^{2}$-cohomology $H_{(2)}^{p}\left(\bar{D}^{*}\right)$ to be $\operatorname{ker}\left(\bar{d}^{p}\right) / \operatorname{clos}\left(\operatorname{im}\left(\bar{d}^{p-1}\right)\right)$, where $\operatorname{clos}\left(\operatorname{im}\left(\bar{d}^{p-1}\right)\right)$ is the closure of the image of $\bar{d}^{p-1}$. Define the $p$-th Laplacian by $\bar{\Delta}_{p}=\left(\bar{d}^{p}\right)^{*} \bar{d}^{p}+\bar{d}^{p-1}\left(\bar{d}^{p-1}\right)^{*}$. By the $L^{2}-$ Hodge-deRham Theorem we get a $G$-equivariant isometric isomorphism $\operatorname{ker}\left(\bar{\Delta}_{p}\right) \cong H_{(2)}^{p}\left(\bar{D}^{*}\right)$. Thus $H_{(2)}^{p}\left(\bar{D}^{*}\right)$ inherits the structure of a Hilbert $\mathcal{N}(G)$-module. Moreover, it turns out to be a finitely generated Hilbert $\mathcal{N}(G)$-module. This can be deduced from the results of [12], where an index already over $C_{r}^{*}(G)$ is defined and the problem of getting finitely generated modules over $C_{r}^{*}(G)$ is treated. Namely, one can deduce from [12] after passing to the group von Neumann algebra, that there are finitely generated Hilbert $\mathcal{N}(G)$-modules $U_{1}, U_{2}, V_{1}$ and $V_{2}$ and Hilbert $\mathcal{N}(G)$-modules $W_{1}$ and $W_{2}$ together with a morphism $v: V_{1} \rightarrow V_{2}$ and isomorphisms of Hilbert $\mathcal{N}(G)$-modules $w: W_{1} \stackrel{\cong}{\rightarrow} W_{2}$, $u_{1}: \bar{D}^{p} \oplus U_{1} \cong V_{1} \oplus W_{1}$ and $u_{2}: \bar{D}^{p} \oplus U_{2} \cong V_{2} \oplus W_{2}$ such that $u_{2} \circ\left(\Delta_{p} \oplus 0\right)=(v \oplus w) \circ u_{1}$. Obviously the kernel of $v$ and hence the kernel of $\bar{\Delta}_{p}$ are finitely generated Hilbert $\mathcal{N}(G)$-modules.

Define the center-valued $L^{2}$-index and the $L^{2}$-index

$$
\begin{aligned}
\operatorname{index}_{\mathcal{N}(G)}^{u}\left(\bar{D}^{*}\right) & :=\sum_{p \geq 0} \operatorname{dim}_{\mathcal{N}(G)}^{u}\left(H_{(2)}^{p}\left(\bar{D}^{*}\right)\right) \in \mathcal{Z}(\mathcal{N}(G)) ; \\
\operatorname{index}_{\mathcal{N}(G)}\left(\bar{D}^{*}\right) & :=\sum_{p \geq 0} \operatorname{dim}_{\mathcal{N}(G)}\left(H_{(2)}^{p}\left(\bar{D}^{*}\right)\right) \in \mathbb{R}
\end{aligned}
$$

The rest of this section is devoted to the proof of Theorem 0.4

Notation 1.9 Denote by $\operatorname{con}(G)_{c f}$ the set of conjugacy classes $(g)$ of elements $g \in G$ such that the set $(g)$ is finite, or, equivalently, the centralizer $C_{g}(g)=\left\{g^{\prime} \in G \mid g^{\prime} g=g g^{\prime}\right\}$ has finite index in $G$. For $c \in \operatorname{con}(G)_{c f}$ let $N_{c}$ be the element $\sum_{g \in c} g \in \mathbb{C} G$. In the sequel $L_{c}$ resp. $L_{g}$ denotes left multiplication with $N_{c}$ resp. $g$ for $c \in \operatorname{con}(G)_{c f}$ resp. $g \in G$.

Notice for the sequel that $N_{c} \in \mathcal{Z}(\mathcal{N}(G))$ and $L_{c}$ is $G$-equivariant and commutes with all $G$ operators.

Lemma 1.10 Consider $a \in \mathcal{Z}(\mathcal{N}(G))$. Then we have $a=0$ if and only if $\operatorname{tr}_{\mathcal{N}(G)}\left(N_{c} a\right)=0$ holds for any $c \in \operatorname{con}_{c f}(G)$.

Proof : Consider $a \in \mathcal{N}(G)=\mathcal{B}\left(l^{2}(G)\right)^{G}$ which belongs to $\mathcal{Z}(\mathcal{N}(G))$. Write $a(e)=\sum_{g \in G} \lambda_{g} \cdot g \in$ $l^{2}(G)$. Since $a R_{g}=R_{g} a$ holds for $g \in G$ and $R_{g}: l^{2}(G) \rightarrow l^{2}(G)$ given by right multiplication with $g \in G$, we get $\lambda_{g}=\lambda_{h g h^{-1}}$ for $g, h \in G$. This implies that $\lambda_{g}=0$ if the conjugacy class $(g)$ is infinite. On easily checks for an element $g$ with finite $(g)$

$$
\lambda_{g}=\operatorname{tr}_{\mathcal{N}(G)}\left(N_{\left(g^{-1}\right)} a\right) .
$$


Lemma 1.11 We get under the conditions above.

$$
\operatorname{tr}_{\mathcal{N}(G)}\left(\operatorname{index}_{\mathcal{N}(G)}^{u}\left(\bar{D}^{*}\right)\right)=\operatorname{index}\left(D^{*}\right) .
$$

Proof : The $L^{2}$-index theorem of Atiyah $[1,(1.1)]$ says

$$
\operatorname{index}_{\mathcal{N}(G)}\left(\bar{D}^{*}\right)=\operatorname{index}\left(D^{*}\right) .
$$

We have

$$
\begin{aligned}
\operatorname{tr}_{\mathcal{N}(G)}\left(\operatorname{index}_{\mathcal{N}(G)}^{u}\left(\bar{D}^{*}\right)\right) & =\operatorname{tr}_{\mathcal{N}(G)}\left(\sum_{p \geq 0}(-1)^{p} \operatorname{dim}_{\mathcal{N}(G)}^{u}\left(H_{(2)}^{p}\left(\bar{D}^{*}\right)\right)\right) \\
& =\sum_{p \geq 0}(-1)^{p} \operatorname{tr}_{\mathcal{N}(G)}\left(\operatorname{dim}_{\mathcal{N}(G)}^{u}\left(H_{(2)}^{p}\left(\bar{D}^{*}\right)\right)\right) \\
& =\sum_{p \geq 0}(-1)^{p} \operatorname{dim}_{\mathcal{N}(G)}\left(H_{(2)}^{p}\left(\bar{D}^{*}\right)\right) \\
& =\operatorname{index}_{\mathcal{N}(G)}\left(\bar{D}^{*}\right) .
\end{aligned}
$$

Next we want to prove

Lemma 1.12 Consider an element $c \in \operatorname{con}(G)_{c f}$ with $c \neq(1)$. Then

$$
\operatorname{tr}_{\mathcal{N}(G)}\left(N_{c} \cdot \operatorname{index}_{\mathcal{N}(G)}^{u}\left(\bar{D}^{*}\right)\right)=0 .
$$

Proof : In the sequel we denote by $\overline{\mathrm{pr}}_{p}: \bar{D}^{p} \rightarrow \bar{D}^{p}$ the projection onto the kernel of the $p$-th Laplacian $\bar{\Delta}_{p}=\left(\bar{d}^{p}\right)^{*} \bar{d}^{p}+\bar{d}^{p-1}\left(\bar{d}^{p-1}\right)^{*}$. By the $L^{2}$-Hodge-deRham Theorem we get a $G$-equivariant isometric isomorphism $\operatorname{im}\left(\overline{\mathrm{pr}}_{p}\right) \stackrel{\cong}{\longrightarrow} H_{(2)}^{p}\left(\bar{D}^{*}\right)$. This implies

$$
\begin{aligned}
& \operatorname{tr}_{\mathcal{N}(G)}\left(N_{c} \cdot \operatorname{index}_{\mathcal{N}(G)}^{u}\left(\bar{D}^{*}\right)\right) \\
& \quad=\sum_{p \geq 0}(-1)^{p} \cdot \operatorname{tr}_{\mathcal{N}(G)}\left(N_{c} \cdot \operatorname{tr}_{\mathcal{N}(G)}^{u}\left(\operatorname{id}: H_{(2)}^{p}\left(\bar{D}^{*}\right) \rightarrow H_{(2)}^{p}\left(\bar{D}^{*}\right)\right)\right) \\
& =\sum_{p \geq 0}(-1)^{p} \cdot \operatorname{tr}_{\mathcal{N}(G)}\left(L_{c}: H_{(2)}^{p}\left(\bar{D}^{*}\right) \rightarrow H_{(2)}^{p}\left(\bar{D}^{*}\right)\right) \\
& =\sum_{p \geq 0}(-1)^{p} \cdot \operatorname{tr}_{\mathcal{N}(G)}\left(L_{c} \circ \overline{\operatorname{pr}}_{p}: \bar{D}^{p} \rightarrow \bar{D}^{p}\right) .
\end{aligned}
$$

The operator $e^{-t \bar{\Delta}_{p}}: \bar{D}^{p} \rightarrow \bar{D}^{p}$ is a bounded $G$-equivariant operator and has a smooth kernel $e^{-t \bar{\Delta}_{p}}(\bar{x}, \bar{y}): \bar{E}_{\bar{x}}^{p} \rightarrow \bar{E}_{\bar{y}}^{p}$ for $\bar{x}, \bar{y} \in \bar{M}$. Thus $e^{-t \bar{\Delta}_{p}}(\omega)$ applied to a section $\omega$ is given at $\bar{y} \in \bar{M}$ by $\int_{\bar{M}} e^{-t \bar{\Delta}_{p}}(\bar{x}, \bar{y})(\omega(\bar{x})) d \operatorname{vol}_{\bar{x}}$. The operator $L_{c} \circ e^{-t \bar{\Delta}_{p}}$ is also a bounded $G$-equivariant operator and has a smooth kernel $\left(L_{c} \circ e^{-t \bar{\Delta}_{p}}\right)(\bar{x}, \bar{y})$ satisfying

$$
\left(L_{c} \circ e^{-t \bar{\Delta}_{p}}\right)(\bar{x}, \bar{y})=\sum_{g \in c} L_{g} \circ e^{-t \bar{\Delta}_{p}}\left(\bar{x}, g^{-1} \bar{y}\right) .
$$


If $\mathcal{F}$ is a fundamental domain for the $G$-action, then [1, Proposition 4.6].

$$
\begin{aligned}
\operatorname{tr}_{\mathcal{N}(G)}\left(L_{c} \circ e^{-t \bar{\Delta}_{p}}\right) & =\int_{\mathcal{F}} \operatorname{tr}_{\mathbb{C}}\left(\left(L_{c} \circ e^{-t \bar{\Delta}_{p}}\right)(\bar{x}, \bar{x})\right) d \operatorname{vol}_{\bar{x}} ; \\
& =\sum_{g \in c} \int_{\mathcal{F}} \operatorname{tr}_{\mathbb{C}}\left(L_{g} \circ e^{-t \bar{\Delta}_{p}}\left(\bar{x}, g^{-1} \bar{x}\right)\right) d \operatorname{vol}_{\bar{x}} .
\end{aligned}
$$

where $\operatorname{tr}_{\mathbb{C}}$ is the trace of an endomorphism of a finite-dimensional complex vector space. Since $M$ is compact, we can find $\epsilon>0$ such that the distance of $\bar{x}$ and $g \bar{x}$ is bounded from below by $\epsilon$ for all $\bar{x} \in \bar{M}$ and $g \in c$. We have

$$
\lim _{t \rightarrow 0} \sup \left\{\left\|e^{-t \bar{\Delta}_{p}}\left(\bar{x}, g^{-1} \bar{x}\right)\right\| \mid \bar{x} \in \mathcal{F}\right\}=0
$$

where $\left\|e^{-t \bar{\Delta}_{p}}\left(\bar{x}, g^{-1} \bar{x}\right)\right\|$ is the operator norm of the linear map $e^{-t \bar{\Delta}_{p}}\left(\bar{x}, g^{-1} \bar{x}\right)$ of finite-dimensional Hilbert spaces. This follows from the finite propagation speed method of [6]. There only the standard Laplacian on 0 -forms is treated, but the proof presented there carries over to the Laplacian $\bar{\Delta}_{p}$ associated to the lift $\bar{D}^{*}$ to the $G$-covering $\bar{M}$ of an elliptic complex $D^{*}$ of differential operators of order 1 on a closed Riemannian manifold $M$ in any dimension $p$. The point is that $\bar{M}$ has bounded geometry, $\bar{\Delta}_{p}$ is essentially selfadjoint and positive so that $\sqrt{\bar{\Delta}_{p}}$ makes sense, and $\frac{\partial^{2}}{\partial t^{2}}+\bar{\Delta}_{p}$ is strictly hyperbolic. Now one applies the results of [6, Section 1] and uses the estimate in [9, page 475], where the special case of $D^{*}$ being the deRham complex is treated.

Since

$$
\left|\operatorname{tr}_{\mathbb{C}}\left(L_{g} \circ e^{-t \bar{\Delta}_{p}}\left(\bar{x}, g^{-1} \bar{x}\right)\right)\right| \leq \operatorname{dim}_{\mathbb{C}}\left(E^{p}\right) \cdot\left\|e^{-t \bar{\Delta}_{p}}\left(\bar{x}, g^{-1} \bar{x}\right)\right\|
$$

and $\mathcal{F}$ is compact, we conclude from (1.14) and (1.15)

$$
\lim _{t \rightarrow 0} \operatorname{tr}_{\mathcal{N}(G)}\left(L_{c} \circ e^{-t \bar{\Delta}_{p}}\right)=0 .
$$

Since the $\operatorname{trace} \operatorname{tr}_{\mathcal{N}(G)}$ is ultraweakly continuous and $\lim _{t \rightarrow \infty} e^{-t \bar{\Delta}_{p}}=\overline{\mathrm{pr}}_{p}$ in the weak topology, we get

$$
\lim _{t \rightarrow \infty} \operatorname{tr}_{\mathcal{N}(G)}\left(L_{c} \circ e^{-t \bar{\Delta}_{p}}\right)=\operatorname{tr}_{\mathcal{N}(G)}\left(L_{c} \circ \overline{\operatorname{pr}}_{p}\right) .
$$

We conclude from (1.13) and (1.17)

$$
\operatorname{tr}_{\mathcal{N}(G)}\left(N_{c} \cdot \operatorname{index}_{\mathcal{N}(G)}^{u}\left(\bar{D}^{*}\right)\right)=\lim _{t \rightarrow \infty} \sum_{p \geq 0}(-1)^{p} \cdot \operatorname{tr}_{\mathcal{N}(G)}\left(L_{c} \circ e^{-t \bar{\Delta}_{p}}\right) .
$$


We have

$$
\begin{aligned}
& \frac{d}{d t} \sum_{p \geq 0}(-1)^{p} \cdot \operatorname{tr}_{\mathcal{N}(G)}\left(L_{c} \circ e^{-t \bar{\Delta}_{p}}: \bar{D}^{p} \rightarrow \bar{D}^{p}\right) \\
& =\sum_{p \geq 0}(-1)^{p} \cdot \operatorname{tr}_{\mathcal{N}(G)}\left(L_{c} \circ \frac{d}{d t} e^{-t \bar{\Delta}_{p}}\right) \\
& =\sum_{p \geq 0}(-1)^{p} \cdot \operatorname{tr}_{\mathcal{N}(G)}\left(L_{c} \circ\left(-\bar{\Delta}_{p}\right) \circ e^{-t \bar{\Delta}_{p}}\right) \\
& =-\sum_{p \geq 0}(-1)^{p} \cdot \operatorname{tr}_{\mathcal{N}(G)}\left(L_{c} \circ \bar{d}^{p-1} \circ\left(\bar{d}^{p-1}\right)^{*} \circ e^{-t \bar{\Delta}_{p}}\right) \\
& -\sum_{p \geq 0}(-1)^{p} \cdot \operatorname{tr}_{\mathcal{N}(G)}\left(L_{c} \circ\left(\bar{d}^{p}\right)^{*} \circ \bar{d}^{p} \circ e^{-t \bar{\Delta}_{p}}\right) \\
& =-\sum_{p \geq 0}(-1)^{p} \cdot \operatorname{tr}_{\mathcal{N}(G)}\left(L_{c} \circ \bar{d}^{p-1} \circ\left(\bar{d}^{p-1}\right)^{*} \circ e^{-\frac{t}{2} \bar{\Delta}_{p}} \circ e^{-\frac{t}{2} \bar{\Delta}_{p}}\right) \\
& -\sum_{p \geq 0}(-1)^{p} \cdot \operatorname{tr}_{\mathcal{N}(G)}\left(L_{c} \circ\left(\bar{d}^{p}\right)^{*} \circ e^{-t \bar{\Delta}_{p+1}} \circ \bar{d}^{p}\right) \\
& =-\sum_{p \geq 0}(-1)^{p} \cdot \operatorname{tr}_{\mathcal{N}(G)}\left(L_{c} \circ e^{-\frac{t}{2} \bar{\Delta}_{p}} \circ \bar{d}^{p-1} \circ\left(\bar{d}^{p-1}\right)^{*} \circ e^{-\frac{t}{2} \bar{\Delta}_{p}}\right) \\
& +\sum_{p \geq 0}(-1)^{p} \cdot \operatorname{tr}_{\mathcal{N}(G)}\left(L_{c} \circ\left(\bar{d}^{p-1}\right)^{*} \circ e^{-t \bar{\Delta}_{p}} \circ \bar{d}^{p-1}\right) \\
& =-\sum_{p \geq 0}(-1)^{p} \cdot \operatorname{tr}_{\mathcal{N}(G)}\left(L_{c} \circ\left(\bar{d}^{p-1}\right)^{*} \circ e^{-\frac{t}{2} \bar{\Delta}_{p}} \circ e^{-\frac{t}{2} \bar{\Delta}_{p}} \circ \bar{d}^{p-1}\right) \\
& +\sum_{p \geq 0}(-1)^{p} \cdot \operatorname{tr}_{\mathcal{N}(G)}\left(L_{c} \circ\left(\bar{d}^{p-1}\right)^{*} \circ e^{-t \bar{\Delta}_{p}} \circ \bar{d}^{p-1}\right) \\
& =-\sum_{p \geq 0}(-1)^{p} \cdot \operatorname{tr}_{\mathcal{N}(G)}\left(L_{c} \circ\left(\bar{d}^{p-1}\right)^{*} \circ e^{-t \bar{\Delta}_{p}} \circ \bar{d}^{p-1}\right) \\
& +\sum_{p \geq 0}(-1)^{p} \cdot \operatorname{tr}_{\mathcal{N}(G)}\left(L_{c} \circ\left(\bar{d}^{p-1}\right)^{*} \circ e^{-t \bar{\Delta}_{p}} \circ \bar{d}^{p-1}\right) \\
& =0 \text {. }
\end{aligned}
$$

Here are some justifications for the calculation above. Recall that $L_{c}$ is a bounded $G$-operator and commutes with any $G$-equivariant operator. We can commute $\operatorname{tr}_{\mathcal{N}(G)}$ and $\frac{d}{d t} \operatorname{since} \operatorname{tr}_{\mathcal{N}(G)}$ is ultraweakly continuous. We conclude $e^{-t \bar{\Delta}_{p+1}} \circ \bar{d}^{p}=\bar{d}^{p} \circ e^{-t \bar{\Delta}_{p}}$ from the fact that $\bar{\Delta}_{p+1} \circ \bar{d}^{p}=\bar{d}^{p} \circ \bar{\Delta}_{p}$ holds on $C^{\infty}\left(\bar{E}^{p-1}\right)$. We have used at several places the typical trace relation $\operatorname{tr}_{\mathcal{N}(G)}(A B)=\operatorname{tr}_{\mathcal{N}(G)}(B A)$ which is in each case justified by [1, section 4]. In order to be able to apply this trace relation we have splitted $e^{-t \bar{\Delta}_{p}}$ into $e^{-\frac{t}{2} \bar{\Delta}_{p}} \circ e^{-\frac{t}{2} \bar{\Delta}_{p}}$ in the calculation above.

Hence $\sum_{p \geq 0}(-1)^{p} \cdot \operatorname{tr}_{\mathcal{N}(G)}\left(L_{c} \circ e^{-t \bar{\Delta}_{p}}: \bar{D}^{p} \rightarrow \bar{D}^{p}\right)$ is independent of $t$ and we conclude from (1.18)

$$
\operatorname{tr}_{\mathcal{N}(G)}\left(N_{c} \cdot \operatorname{index}_{\mathcal{N}(G)}^{u}\left(\bar{D}^{*}\right)\right)=\lim _{t \rightarrow 0} \sum_{p \geq 0}(-1)^{p} \cdot \operatorname{tr}_{\mathcal{N}(G)}\left(L_{c} \circ e^{-t \bar{\Delta}_{p}}: \bar{D}^{p} \rightarrow \bar{D}^{p}\right)
$$

Now Lemma 1.12 follows from (1.16) (1.20).

Finally Theorem 0.4 follows from Lemma 1.10, Lemma 1.11 and Lemma 1.12. 


\section{Modules over a category}

In this section we recall some facts about modules over the category $\operatorname{Sub}=\operatorname{Sub}(G ; \mathcal{F}(X))$ for a proper $G$-CW-complex $X$ as far as needed here. For more information about modules over a category we refer to [10].

Let $\mathrm{Sub}:=\operatorname{Sub}(G ; \mathcal{F}(X))$ be the following category. Objects are the elements of the set $\mathcal{F}(X)$ of subgroups $H \subset G$, for which $X^{H} \neq \emptyset$. For two finite subgroups $H$ and $K$ in $\mathcal{F}(X)$ denote by $\operatorname{conhom}_{G}(H, K)$ the set of group homomorphisms $f: H \rightarrow K$, for which there exists an element $g \in G$ with $g H^{-1} \subset K$ such that $f$ is given by conjugation with $g$, i.e. $f=c(g): H \rightarrow K, \quad h \mapsto g h g^{-1}$. Notice that $c(g)=c\left(g^{\prime}\right)$ holds for two elements $g, g^{\prime} \in G$ with $g H g^{-1} \subset K$ and $g^{\prime} H\left(g^{\prime}\right)^{-1} \subset K$ if and only if $g^{-1} g^{\prime}$ lies in the centralizer $C_{G} H=\{g \in G \mid g h=h g$ for all $h \in H\}$ of $H$ in $G$. The group of inner automorphisms of $K$ acts on $\operatorname{conhom}_{G}(H, K)$ from the left by composition. Define the set of morphisms mor sub $(H, K)$ by $\operatorname{Inn}(K) \backslash \operatorname{conhom}_{G}(H, K)$. Let $N_{G} H$ be the normalizer $\{g \in G \mid$ $\left.g H g^{-1}=H\right\}$ of $H$. Define $H \cdot C_{G} H=\left\{h \cdot g \mid h \in H, g \in C_{G} H\right\}$. This is a normal subgroup of $N_{G} H$ and we define $W_{G} H:=N_{G} H /\left(H \cdot C_{G} H\right)$. One easily checks that $W_{G} H$ is a finite group and that there is an isomorphism from $W_{G} H$ to autsub $(H)$ which sends $g\left(H \cdot C_{G} H\right) \in W_{G} H$ to the automorphism of $H$ represented by $c(g): H \rightarrow H$. Notice that there is a morphism from $H$ to $K$ if and only if $H$ is subconjugated to $K$. There is an isomorphism from $H$ to $K$ if and only if $H$ and $K$ are conjugated. The category Sub is a so called EI-category, i.e. any endomorphism in Sub is an isomorphism.

Let $R$ be a commutative associative ring with unit. A covariant resp. contravariant $R$ Sub-module $M$ is a covariant resp. contravariant functor from $S u b$ to the category of $R$-modules. Morphisms are natural transformations. The structure of an abelian category on the category of $R$-modules carries over to the category of $R S u b$-modules. In particular the notion of a projective $R S u b$-module is defined. Given a contravariant $R S u b$-module $M$ and a covariant $R S u b$-module $N$, one can define a $R$-module, their tensor product over Sub

$$
M \otimes_{R \text { Sub }} N=\oplus_{H \in \mathcal{F}(X)} M(H) \otimes_{R} N(H) / \sim,
$$

where $\sim$ is the typical tensor relation $m f \otimes n=m \otimes f n$, i.e. for each morphism $f: H \rightarrow K$ in Sub, $m \in M(K)$ and $n \in N(H)$ we introduce the relation $M(f)(m) \otimes n-m \otimes N(f)(n)=0$.

Given a left $R\left[W_{G} H\right]$-module $N$ for $H \in \mathcal{F}(X)$, define a covariant $R$ Sub-module $E_{H} M$ by

$$
\left(E_{H} M\right)(K):=R \operatorname{mor}_{\text {Sub }}(H, K) \otimes_{R\left[W_{G} H\right]} N \quad \text { for } K \subset G,|K|<\infty,
$$

where $R$ morsub $_{\mathrm{Sub}}(H, K)$ is the free $R$-module generated by the set mor $\operatorname{sub}(H, K)$. Given a covariant $R$ Sub-module $M$ and $H \in \mathcal{F}(X)$, define $M(H)_{s}$ to be the left $R$-submodule of $M(H)$, which is spanned by the images of all $R$-maps $M(f): M(K) \rightarrow M(H)$, where $f$ runs through all morphisms $f: K \rightarrow H$ in Sub, which have $H$ as target and are not isomorphisms. Obviously $M(H)_{s}$ is an $R\left[W_{G} H\right]$-submodule of $M(H)$. Define a left $R\left[W_{G} H\right]$-module $S_{H} M$ by

$$
S_{H} M:=M(H) / M(H)_{s} .
$$

Both functors $E_{H}$ and $S_{H}$ respect direct sums and the property finitely generated and the property projective. Given a left $R\left[W_{G} H\right]$-module $M, S_{K} \circ E_{H} M$ is $M$, if $H=K$ and is 0 , if $H$ and $K$ are not conjugated in $G$.

Let $M$ be a covariant $R S$ ub-module. We want to check whether it is projective or not. A necessary (but not sufficient) condition is that $S_{H} M$ is a projective $R\left[W_{G} H\right]$-module. Assume that $S_{H} M$ is $R\left[W_{G} H\right]$-projective for all objects $H$ in Sub. We can choose a $R\left[W_{G} H\right]$-splitting $\sigma_{H}: S_{H} M \rightarrow M(H)$ of the canonical projection $M(H) \rightarrow S_{H} M=M(H) / M(H)_{s}$. For a finite subgroup $H \subset G$ define the morphism of covariant $R$ Sub-modules

$$
i_{H} M: E_{H}(M(H)) \rightarrow M
$$


by $\left(i_{H} M\right)(K)\left((f: H \rightarrow K) \otimes_{R\left[W_{G} H\right]} m\right)=M(f)(m)$. We obtain after a choice of representatives $H \in(H)$ for any conjugacy class $(H)$ of subgroups $H \in \mathcal{F}(X)$ a morphism of covariant $R$ Sub-modules

$$
T: \oplus_{(H), H \in \mathcal{F}(X)} E_{H} S_{H} M \stackrel{\oplus_{(H), H \in \mathcal{F}(X)} E_{H}\left(\sigma_{H}\right)}{\longrightarrow} \oplus_{(H), H \in \mathcal{F}(X)} E_{H}(M(H)) \stackrel{\oplus_{(H), H \in \mathcal{F}(X)} i_{H} M}{\longrightarrow} M .
$$

We get as a special case of [11, Theorem 2.11]

Theorem 2.4 The morphism $T$ is always surjective. It is bijective if and only if $M$ is a projective RSub-module.

\section{Some representation theory for finite groups}

Denote for a finite group $H$ by $\operatorname{Rep}_{\mathbb{Q}}(H)$ resp. $\operatorname{Rep}_{\mathbb{C}}(H)$ the ring of finite dimensional $H$ representations over the field $\mathbb{Q}$ resp. $\mathbb{C}$. Recall for the sequel that these are finitely generated free abelian groups. Given an inclusion of finite groups $H \subset G$, we denote by $\operatorname{ind}_{H}^{G}: \operatorname{Rep}_{\mathbb{Q}}(H) \rightarrow \operatorname{Rep}_{\mathbb{Q}}(G)$ and $\operatorname{res}_{G}^{H}: \operatorname{Rep}_{\mathbb{Q}}(G) \rightarrow \operatorname{Rep}_{\mathbb{Q}}(H)$ the induction and restriction homomorphism and similar for $R \otimes_{\mathbb{Z}} \operatorname{Rep}_{\mathbb{Q}}, \operatorname{Rep}_{\mathbb{C}}$ and $R \otimes_{\mathbb{Z}} \operatorname{Rep}_{\mathbb{C}}$ for a commutative ring $R$ with $\mathbb{Z} \subset R$. Let $\operatorname{con}_{\mathbb{Q}}(H)$ be the set of $\mathbb{Q}$-conjugacy classes of elements in $H$, where $h$ and $h^{\prime}$ are called $\mathbb{Q}$-conjugated if the cyclic subgroups $\langle h\rangle$ and $\left\langle h^{\prime}\right\rangle$ are conjugated in $G$. Let $\operatorname{con}(G)$ be the set of conjugacy classes of elements in $G$. Denote by $\operatorname{class}_{\mathbb{Q}}(H)$ resp. $\operatorname{class}_{\mathbb{C}}(H)$ the rational resp. complex vector space of functions $\operatorname{con}_{\mathbb{Q}}(H) \rightarrow \mathbb{Q}$ resp. $\operatorname{con}(G) \rightarrow \mathbb{C}$. Character theory yields isomorphisms [15, page 68 and Theorem 29 on page 102]

$$
\begin{aligned}
& \chi_{\mathbb{Q}}: \mathbb{Q} \otimes_{\mathbb{Z}} \operatorname{Rep}_{\mathbb{Q}}(H) \stackrel{\cong}{\operatorname{class}_{\mathbb{Q}}}(H) ; \\
& \chi_{\mathbb{C}}: \mathbb{C} \otimes_{\mathbb{Z}} \operatorname{Rep}_{\mathbb{C}}(H) \stackrel{\cong}{\operatorname{class}_{\mathbb{C}}}(H) \text {. }
\end{aligned}
$$

For a finite cyclic group $C$ denote by $\theta_{C} \in \mathbb{Q} \otimes_{\mathbb{Z}} \operatorname{Rep}_{\mathbb{Q}}(C)$ the element whose character $\chi_{\mathbb{Q}}\left(\theta_{C}\right)$ sends $c \in C$ to 1 , if $c$ generates $C$, and to 0 otherwise.

Let $C \subset H$ be a cyclic subgroup of the finite group $H$. Then we get for $h \in H$

$$
\frac{1}{[H: C]} \cdot \chi_{\mathbb{Q}}\left(\operatorname{ind}_{C}^{H} \theta_{C}\right)(h)=\frac{1}{[H: C]} \cdot \frac{1}{|C|} \cdot \sum_{l \in H, l^{-1} h l \in C} \chi_{\mathbb{Q}}\left(\theta_{C}\right)\left(l^{-1} h l\right)=\frac{1}{|H|} \cdot \sum_{l \in H,\left\langle l^{-1} h l\right\rangle=C} 1 .
$$

Denote by $[\mathbb{Q}] \in \operatorname{Rep}_{\mathbb{Q}}(H)$ the class of the trivial $H$-representation $\mathbb{Q}$. Notice that $\chi_{\mathbb{Q}}([\mathbb{Q}])$ is the constant function with values 1 . We get in $\mathbb{Q} \otimes_{\mathbb{Z}} \operatorname{Rep}_{\mathbb{Q}}(H)$

$$
1 \otimes_{\mathbb{Z}}[\mathbb{Q}]=\sum_{C \subset H, C \text { cyclic }} \frac{1}{[H: C]} \cdot \operatorname{ind}_{C}^{H} \theta_{C},
$$

since for any $l \in H$ and $h \in H$ there is precisely one cyclic subgroup $C \subset H$ with $C=\left\langle l^{-1} h l\right\rangle$ and $\chi_{\mathbb{Q}}$ is bijective. In particular we get for a finite cyclic group $C$ in $\mathbb{Q} \otimes_{\mathbb{Z}} \operatorname{Rep}_{\mathbb{Q}}(C)$

$$
\theta_{C}=1 \otimes_{\mathbb{Z}}[\mathbb{Q}]-\sum_{D \subset C, D \neq C} \frac{1}{[C: D]} \cdot \operatorname{ind}_{D}^{C} \theta_{D}
$$

Now one easily checks by induction over the order of the finite cyclic subgroup $C$ that the element $\theta_{C}$ satisfies

$$
\theta_{C} \in \mathbb{Z}\left[\frac{1}{|C|}\right] \otimes_{\mathbb{Z}} \operatorname{Rep}_{\mathbb{Q}}(C) .
$$


Obviously $\theta_{C}$ is an idempotent in $\mathbb{Z}\left[\frac{1}{|C|}\right] \otimes_{\mathbb{Z}} \operatorname{Rep}_{\mathbb{Q}}(C)$. By the obvious change of rings homomorphism, $\mathbb{Z}\left[\frac{1}{|C|}\right] \otimes_{\mathbb{Z}} \operatorname{Rep}_{\mathbb{C}}(C)$ becomes a $\mathbb{Z}\left[\frac{1}{|C|}\right] \otimes_{\mathbb{Z}} \operatorname{Rep}_{\mathbb{Q}}(C)$-module. Hence multiplication with $\theta_{C}$ defines an idempotent endomorphism

$$
\theta_{C}: \mathbb{Z}\left[\frac{1}{|C|}\right] \otimes_{\mathbb{Z}} \operatorname{Rep}_{\mathbb{C}}(C) \rightarrow \mathbb{Z}\left[\frac{1}{|C|}\right] \otimes_{\mathbb{Z}} \operatorname{Rep}_{\mathbb{C}}(C) .
$$

It is natural with respect to group automorphisms of $C$, since $\theta_{C}$ is invariant under group automorphisms of $C$.

Lemma $3.4 \quad$ (a) For a finite group $H$ the map

$$
\oplus_{C \subset H, C \text { cyclic }} \operatorname{ind}_{C}^{H}: \oplus_{C \subset H, C \text { cyclic }} \mathbb{Z}\left[\frac{1}{|H|}\right] \otimes_{\mathbb{Z}} \operatorname{Rep}_{\mathbb{C}}(C) \rightarrow \mathbb{Z}\left[\frac{1}{|H|}\right] \otimes_{\mathbb{Z}} \operatorname{Rep}_{\mathbb{C}}(H)
$$

is surjective;

(b) Let $C$ be a finite cyclic group. Then the image resp. cokernel of

$$
\oplus_{D \subset C, D \neq C} \operatorname{ind}_{D}^{C}: \oplus_{D \subset C, D \neq C} \mathbb{Z}\left[\frac{1}{|C|}\right] \otimes_{\mathbb{Z}} \operatorname{Rep}_{\mathbb{C}}(D) \rightarrow \mathbb{Z}\left[\frac{1}{|C|}\right] \otimes_{\mathbb{Z}} \operatorname{Rep}_{\mathbb{C}}(C)
$$

is equal resp. isomorphic to the kernel resp. image of the idempotent endomorphism

$$
\theta_{C}: \mathbb{Z}\left[\frac{1}{|C|}\right] \otimes_{\mathbb{Z}} \operatorname{Rep}_{\mathbb{C}}(C) \rightarrow \mathbb{Z}\left[\frac{1}{|C|}\right] \otimes_{\mathbb{Z}} \operatorname{Rep}_{\mathbb{C}}(C)
$$

(c) Let $C$ be a finite cyclic group. The image of the idempotent endomorphism

$$
\theta_{C}: \mathbb{Z}\left[\frac{1}{|C|}\right] \otimes_{\mathbb{Z}} \operatorname{Rep}_{\mathbb{C}}(C) \rightarrow \mathbb{Z}\left[\frac{1}{|C|}\right] \otimes_{\mathbb{Z}} \operatorname{Rep}_{\mathbb{C}}(C)
$$

is a projective $\mathbb{Z}\left[\frac{1}{|C|}\right][\operatorname{aut}(C)]$-module, where the aut $(C)$-operation comes from the obvious $\operatorname{aut}(C)$-operation on $C$ and induction.

Proof : (a) follows from the following calculation for $x \in \mathbb{Z}\left[\frac{1}{|H|}\right] \otimes_{\mathbb{Z}} \operatorname{Rep}_{\mathbb{C}}(H)$ based on (3.1)

$$
x=\left(1 \otimes_{\mathbb{Z}}[\mathbb{Q}]\right) \cdot x=\left(\sum_{C \subset H, C \text { cyclic }} \frac{1}{[H: C]} \cdot \operatorname{ind}_{C}^{H} \theta_{C}\right) \cdot x=\sum_{C \subset H, C \text { cyclic }} \frac{1}{[H: C]} \cdot \operatorname{ind}_{C}^{H}\left(\theta_{C} \cdot \operatorname{res}_{H}^{C} x\right) .
$$

(b) follows from the following two calculations based on (3.2) for $x \in \mathbb{Z}\left[\frac{1}{|C|}\right] \otimes_{\mathbb{Z}} \operatorname{Rep}_{\mathbb{C}}(H)$

$$
\begin{aligned}
x-\theta_{C} \cdot x & =\left(1 \otimes[\mathbb{Q}]-\theta_{C}\right) \cdot x \\
& =\left(\sum_{D \subset C, D \neq C} \frac{1}{[C: D]} \cdot \operatorname{ind}_{D}^{C} \theta_{D}\right) \cdot x \\
& =\sum_{D \subset C, D \neq C} \frac{1}{[C: D]} \cdot \operatorname{ind}_{D}^{C}\left(\theta_{D} \cdot \operatorname{res}_{C}^{D} x\right)
\end{aligned}
$$

and for $D \subset C, D \neq C$ and $y \in \mathbb{Z}\left[\frac{1}{|C|}\right] \otimes_{\mathbb{Z}} \operatorname{Rep}_{\mathbb{C}}(D)$

$$
\theta_{C} \cdot \operatorname{ind}_{D}^{C} y=\operatorname{ind}_{D}^{C}\left(\operatorname{res}_{C}^{D} \theta_{C} \cdot y\right)=\operatorname{ind}_{D}^{C}(0 \cdot y)=0 .
$$


(c) Put $\Lambda=\mathbb{Z}\left[\frac{1}{|C|}\right]$. Let $C_{p}$ be the $p$-Sylow subgroup of $C$ for a prime $p$. There are canonical isomorphisms

$$
\begin{aligned}
C & \cong \prod_{p} C_{p} ; \\
\operatorname{aut}(C) & \cong \prod_{p} \operatorname{aut}\left(C_{p}\right) ; \\
P: \otimes_{p} \operatorname{Rep}_{\mathbb{C}}\left(C_{p}\right) & \cong \operatorname{Rep}_{\mathbb{C}}(C),
\end{aligned}
$$

where $p$ runs through the prime numbers diving $|C|$. The isomorphism $P$ assigns to $\otimes_{p}\left[V_{p}\right]$ for $C_{p^{-}}$ representations $V_{p}$ the class of the $C$-representation $\otimes_{p} V_{p}$ with the factorwise action of aut $(C)=$ $\prod_{p}$ aut $\left(C_{p}\right)$. The following diagram commutes

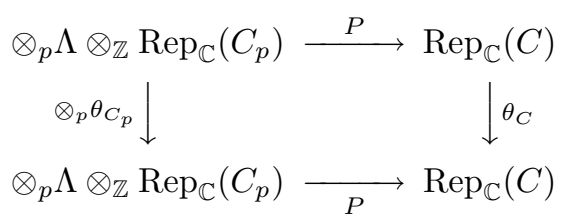

Thus we obtain an isomorphism of $\Lambda[\operatorname{aut}(C)]$-modules

$$
\otimes_{p} \operatorname{im}\left(\theta_{C_{p}}\right) \stackrel{\cong}{\cong} \operatorname{im}\left(\theta_{C}\right),
$$

where aut $(C)=\prod_{p}$ aut $\left(C_{p}\right)$ acts factorwise on the source. Hence the claim for $C$ follows if we know it for $C_{p}$ for all primes $p$. Therefore it remains to treat the case $C=\mathbb{Z} / p^{n}$ for some prime number $p$ and positive integer $n$. Notice that then $\Lambda=\mathbb{Z}\left[\frac{1}{p}\right]$.

In the sequel we abbreviate $A(n)=\operatorname{aut}\left(\mathbb{Z} / p^{n}\right)$. This is isomorphic to multiplicative group of units $\mathbb{Z} / p^{n \times}$ in $\mathbb{Z} / p^{n}$ and hence an abelian group of order $p^{n-1} \cdot(p-1)$. Denote by $A(n)_{p}$ the $p$-Sylow subgroup and by $A(n)_{p}^{\prime}$ the subgroup $\left\{a \in A(n) \mid a^{p-1}=1\right\}$ which is cyclic of order $(p-1)$. We get a canoncial isomorphism

$$
A(n) \cong A(n)_{p} \times A(n)_{p}^{\prime}
$$

Notice that $\mathbb{Z} / p^{n}$ has precisely one subgroup of order $p^{m}$ for $0 \leq m \leq n$ which will be denoted by $\mathbb{Z} / p^{m}$. These subgroups are characteristic and hence restriction to these subgroups yields homomorphisms $A(n) \rightarrow A(n-1) \rightarrow \ldots \rightarrow A(1)$. They induce epimorphisms $A(m)_{p} \rightarrow A(m-1)_{p}$ and isomorphisms $A(m)_{p}^{\prime} \stackrel{\cong}{\longrightarrow} A(m-1)_{p}^{\prime}$. Using these isomorphisms we will identify

$$
A(n)_{p}^{\prime}=A(n-1)_{p}^{\prime}=\ldots=A(1)_{p}^{\prime}=\mathbb{Z} / p^{\times} .
$$

Thus we get canonical decompositions

$$
A(n)=A(n)_{p} \times \mathbb{Z} / p^{\times} .
$$

Let $M$ be a $\Lambda[A(n)]$-module. Let res $M$ be the $\Lambda\left[\mathbb{Z} / p^{\times}\right]$-module obtained by restriction. The following maps are $\Lambda[A(n)]$-homomorphisms

$$
\begin{array}{rlrl}
q: \Lambda\left[A(n)_{p}\right] \otimes_{\Lambda} \operatorname{res} M \rightarrow M, & a \otimes m & \mapsto a m ; \\
s: M \rightarrow \Lambda\left[A(n)_{p}\right] \otimes_{\Lambda} \operatorname{res} M, & m \mapsto \frac{1}{\left|A(n)_{p}\right|} \cdot \sum_{a \in A(n)_{p}} a \otimes a^{-1} m,
\end{array}
$$


where $A(n)=A(n)_{p} \times \mathbb{Z} / p^{\times}$acts factorwise on $\Lambda\left[A(n)_{p}\right] \otimes_{\Lambda}$ res $M$. They satisfy $q \circ s=$ id. Obviously $\Lambda\left[A(n)_{p}\right] \otimes_{\Lambda}$ res $M$ is $\Lambda[A(n)]$-projective if res $M$ is $\Lambda\left[\mathbb{Z} / p^{\times}\right]$-projective. This shows that $M$ is $\Lambda[A(n)]$ projective if its restriction res $M$ to a $\Lambda\left[\mathbb{Z} / p^{\times}\right]$-module is projective. Therefore it suffices to show that $\operatorname{im}\left(\theta_{C}\right)$ is $\Lambda\left[\mathbb{Z} / p^{\times}\right]$-projective.

The composition of the induction homomorphism $\operatorname{Rep}_{\mathbb{C}}\left(\mathbb{Z} / p^{n-1}\right) \rightarrow \operatorname{Rep}_{\mathbb{C}}\left(\mathbb{Z} / p^{n}\right)$ with the restriction homomorphism $\operatorname{Rep}_{\mathbb{C}}\left(\mathbb{Z} / p^{n}\right) \rightarrow \operatorname{Rep}_{\mathbb{C}}\left(\mathbb{Z} / p^{n-1}\right)$ is $p \cdot$ id $: \operatorname{Rep}_{\mathbb{C}}\left(\mathbb{Z} / p^{n-1}\right) \rightarrow \operatorname{Rep}_{\mathbb{C}}\left(\mathbb{Z} / p^{n-1}\right)$. We conclude from Lemma 3.4 (b) that the $\Lambda\left[\mathbb{Z} / p^{\times}\right]$-module $\operatorname{im}\left(\theta_{C}\right)$ is isomorphic to the kernel of the surjective restriction homomorphism res : $\Lambda \otimes_{\mathbb{Z}} \operatorname{Rep}_{\mathbb{C}}\left(\mathbb{Z} / p^{n}\right) \rightarrow \Lambda \otimes_{\mathbb{Z}} \operatorname{Rep}_{\mathbb{C}}\left(\mathbb{Z} / p^{n-1}\right)$. Hence there is an exact sequence of $\Lambda\left[\mathbb{Z} / p^{\times}\right]$-modules

$$
0 \rightarrow \operatorname{im}\left(\theta_{c}\right) \rightarrow \Lambda \otimes_{\mathbb{Z}} \operatorname{Rep}_{\mathbb{C}}\left(\mathbb{Z} / p^{n}\right) \rightarrow \Lambda \otimes_{\mathbb{Z}} \operatorname{Rep}_{\mathbb{C}}\left(\mathbb{Z} / p^{n-1}\right) \rightarrow 0 .
$$

It induces an exact sequence of $\Lambda\left[\mathbb{Z} / p^{\times}\right]$-modules

$$
\begin{aligned}
0 & \rightarrow \operatorname{im}\left(\theta_{c}\right) \rightarrow \operatorname{ker}\left(\Lambda \otimes_{\mathbb{Z}} \operatorname{Rep}_{\mathbb{C}}\left(\mathbb{Z} / p^{n}\right) \rightarrow \Lambda \otimes_{\mathbb{Z}} \operatorname{Rep}_{\mathbb{C}}(\{1\})\right) \\
& \rightarrow \operatorname{ker}\left(\Lambda \otimes_{\mathbb{Z}} \operatorname{Rep}_{\mathbb{C}}\left(\mathbb{Z} / p^{n-1}\right) \rightarrow \Lambda \otimes_{\mathbb{Z}} \operatorname{Rep}_{\mathbb{C}}(\{1\})\right) \rightarrow 0 .
\end{aligned}
$$

Hence it suffices to show that the $\Lambda\left[\mathbb{Z} / p^{\times}\right]$-module $\operatorname{ker}\left(\Lambda \otimes_{\mathbb{Z}} \operatorname{Rep}_{\mathbb{C}}\left(\mathbb{Z} / p^{m}\right) \rightarrow \Lambda \otimes_{\mathbb{Z}} \operatorname{Rep}_{\mathbb{C}}(\{1\})\right)$ is projective for $m=1,2 \ldots n$.

Recall that $\mathbb{Z} / p^{\times}$is a subgroup of $A(m)=\operatorname{aut}\left(\mathbb{Z} / p^{m}\right)$ and thus acts on $\mathbb{Z} / p^{m}-\{\overline{0}\}$ in the obvious way. Denote for $k \in \mathbb{Z}$ by $\mathbb{C}_{k}$ the one-dimensional $\mathbb{Z} / p^{m}$-representation for which $\bar{b} \in \mathbb{Z} / p^{m}$ acts by multiplication with $\exp (2 \pi i k b)$. We obtain a $\Lambda\left[\mathbb{Z} / p^{\times}\right]$-homomorphism

$$
Q: \Lambda\left[\mathbb{Z} / p^{m}-\{0\}\right] \rightarrow \operatorname{ker}\left(\Lambda \otimes_{\mathbb{Z}} \operatorname{Rep}_{\mathbb{C}}\left(\mathbb{Z} / p^{m}\right) \rightarrow \Lambda \otimes_{\mathbb{Z}} \operatorname{Rep}_{\mathbb{C}}(\{1\})\right)
$$

by sending $\bar{k}$ to $\left[\mathbb{C}_{k}\right]-\frac{1}{p^{m}} \cdot\left[\mathbb{C}\left[\mathbb{Z} / p^{m}\right]\right]$. This is the composition of the inclusion $\Lambda\left[\mathbb{Z} / p^{m}-\overline{0}\right] \rightarrow$ $\Lambda\left[\mathbb{Z} / p^{m}\right]$, the isomorphism $\Lambda\left[\mathbb{Z} / p^{m}\right] \rightarrow \Lambda \otimes_{\mathbb{Z}} \operatorname{Rep}\left(\mathbb{Z} / p^{m}\right)$ sending $\bar{k}$ to $\left[\mathbb{C}_{k}\right]$ and the split epimorphism $\Lambda \otimes_{\mathbb{Z}} \operatorname{Rep}\left(\mathbb{Z} / p^{m}\right) \rightarrow \operatorname{ker}\left(\Lambda \otimes_{\mathbb{Z}} \operatorname{Rep}_{\mathbb{C}}\left(\mathbb{Z} / p^{m}\right) \rightarrow \Lambda \otimes_{\mathbb{Z}} \operatorname{Rep} \mathbb{C}_{\mathbb{C}}(\{1\})\right)$ sending $[V]$ to $[V]-\frac{\operatorname{dim}(V)}{p^{m}} \cdot\left[\mathbb{C}\left[\mathbb{Z} / p^{m}\right]\right]$. One easily checks that $Q$ is an isomorphism of $\Lambda\left[\mathbb{Z} / p^{\times}\right]$-modules. Hence it remains to show that $\mathbb{Z} / p^{\times}$acts freely on $\mathbb{Z} / p^{m}-\{\overline{0}\}$ because then $\Lambda\left[\mathbb{Z} / p^{m}-\{\overline{0}\}\right]$ is a free $\Lambda\left[\mathbb{Z} / p^{\times}\right]$-module.

Consider $x \in \mathbb{Z} / p^{m}$ with $x \neq \overline{0}$. We have to show for $a \in \mathbb{Z} / p^{\times}=A(m)_{p}^{\prime} \subset A(m)$ that $a(x)=x$ implies $a=$ id. Since $x$ is non-zero, $x$ generates a cyclic subgroup $\mathbb{Z} / p^{l}$ for some $l \in\{1,2, \ldots m\}$. Then $a \in A(m)$ restricted to $A(l)$ is an automorphism $\mathbb{Z} / p^{l} \rightarrow \mathbb{Z} / p^{l}$ which sends a generator to itself. Hence this automorphism of $\mathbb{Z} / p^{l}$ is the identity. This implies that $a$ is the identity in $A(l)_{p}^{\prime}=\mathbb{Z} / p^{\times}$. This finishes the proof of Lemma 3.4.

The next result is analogous to [11, Lemma 7.4] but we have to go through its proof again because here we want to invert only the orders of finite subgroups of $G$, whereas in [11] we have considered everything over $\mathbb{Q}$.

Theorem 3.5 Let $G$ be a group and $\Lambda=\Lambda^{G}(X)$ as defined in (0.5). Consider the covariant $\Lambda$ Submodule $\Lambda \otimes_{\mathbb{Z}} \operatorname{Rep}($ ? $)$ which sends a finite subgroup group $H \subset G$ to $\Lambda \otimes_{\mathbb{Z}} \operatorname{Rep}_{\mathbb{C}}(H)$. Then

(a) $S_{H} \Lambda \otimes_{\mathbb{Z}} \operatorname{Rep}_{\mathbb{C}}($ ?) is trivial if the finite subgroup $H \subset G$ is not cyclic.

For a finite cyclic subgroup $C \subset G$, the $\Lambda\left[W_{G} C\right]$-module $S_{C} \Lambda \otimes_{\mathbb{Z}} \operatorname{Rep}_{\mathbb{C}}($ ?) is isomorphic to the image of the idempotent $\Lambda\left[W_{G} C\right]$-homomorphism

$$
\theta_{C}: \Lambda \otimes_{\mathbb{Z}} \operatorname{Rep}_{\mathbb{C}}(C) \rightarrow \Lambda \otimes_{\mathbb{Z}} \operatorname{Rep}_{\mathbb{C}}(C) .
$$

The isomorphism is given by the composition of the obvious inclusion $\operatorname{im}\left(\theta_{C}\right) \rightarrow \operatorname{Rep}_{\mathbb{C}}(C)$ with the obvious projection $\operatorname{Rep}_{\mathbb{C}}(C) \rightarrow S_{C} \Lambda \otimes_{\mathbb{Z}} \operatorname{Rep}_{\mathbb{C}}(?)$; 
(b) $\Lambda \otimes_{\mathbb{Z}} \operatorname{Rep}_{\mathbb{C}}($ ?) is a projective $\Lambda$ Sub-module;

(c) Let $M$ be a contravariant $\Lambda \mathrm{Sub}$-module. There is a natural isomorphism of $\Lambda$-modules

$$
\begin{array}{r}
\oplus_{(C), C \text { cyclic }, C \in \mathcal{F}(X)} M(C) \otimes_{\Lambda\left[W_{G} C\right]} \operatorname{im}\left(\theta_{C}: \Lambda \otimes_{\mathbb{Z}} \operatorname{Rep}_{\mathbb{C}}(C) \rightarrow \Lambda \otimes_{\mathbb{Z}} \operatorname{Rep}_{\mathbb{C}}(C)\right) \\
\cong \quad M \otimes_{\Lambda \text { Sub }} \Lambda \otimes_{\mathbb{Z}} \operatorname{Rep}_{\mathbb{C}}(?) ;
\end{array}
$$

(d) $\Lambda \otimes_{\mathbb{Z}} \operatorname{Rep}_{\mathbb{C}}(H)$ is a flat $\Lambda$ Sub-module, i.e. for an exact sequence $0 \rightarrow M_{0} \rightarrow M_{1} \rightarrow M_{2} \rightarrow 0$ of contravariant $\Lambda$ Sub-modules the induced sequence of $R$-modules $0 \rightarrow M_{0} \otimes_{\Lambda \text { Sub }} \Lambda \otimes_{\mathbb{Z}} \operatorname{Rep}_{\mathbb{C}}($ ?) $\rightarrow$ $M_{1} \otimes_{\Lambda \text { Sub }} \Lambda \otimes_{\mathbb{Z}} \operatorname{Rep}_{\mathbb{C}}(?) \rightarrow M_{2} \otimes_{\Lambda \text { Sub }} \Lambda \otimes_{\mathbb{Z}} \operatorname{Rep}_{\mathbb{C}}(?) \rightarrow 0$ is exact.

Proof : (a) We conclude from Lemma 3.4 (a) that $S_{H} \Lambda \otimes_{\mathbb{Z}} \operatorname{Rep}_{\mathbb{C}}($ ?) is trivial if $H$ is not cyclic. If $\overline{\overline{H=C}}$ for a finite cyclic subgroup $C \subset G$, the assertion follows from Lemma 3.4 (b).

(b) Notice that $N_{G} H / C_{G} H$ is a subgroup of aut $(H)$ and all $W_{G} H$-operations are induced by the obvious aut $(H)$-operations. We conclude from Lemma 3.4 (c) and assertion (a) that $S_{H} \Lambda \otimes_{\mathbb{Z}} \operatorname{Rep_{\mathbb {C}}}($ ?) is a projective $\Lambda\left[W_{G} H\right]$-module for all $H \in \mathcal{F}(X)$. Because of Theorem 2.4 it suffices to show for the morphism $T$ defined in (2.3) that $T(K)$ is injective for any given element $K \in \mathcal{F}(X)$.

Consider an element $u$ in the kernel of $T(K)$. Put $J(H)=$ morsub $_{\text {sub }}(H, K) /\left(W_{G} H\right)$ for $H \in \mathcal{F}(X)$ and put $I=\{(H) \mid H \in \mathcal{F}(X)\}$. Choose for any $(H) \in I$ a representative $H \in(H)$. Then fix for any element $\bar{f} \in J(H)$ a representative $f: H \rightarrow K$ in $\operatorname{mor}_{\mathrm{Sub}}(H, K)$. For the remainder of the proof of assertion (b) we abbreviate $L(?):=\Lambda \otimes_{\mathbb{Z}} \operatorname{Rep}_{\mathbb{C}}\left(\right.$ ?). We can find elements $x_{H, f} \in S_{H} L$ for $(H) \in I$ and $\bar{f} \in J(H)$ such that only finitely many of the $x_{H, f}$-s are different from zero and $u$ can be written as

$$
u=\sum_{(H) \in I} \sum_{\bar{f} \in J(H)}(f: H \rightarrow K) \otimes_{\Lambda\left[W_{G} H\right]} x_{H, f} .
$$

We want to show that all elements $x_{H, f}$ are zero. Suppose that this is not the case. Let $\left(H_{0}\right)$ be maximal among those elements $(H) \in I$ for which there is $\bar{f} \in J(H)$ with $x_{H, f} \neq 0$, i.e. if for $(H) \in I$ the element $x_{H, f}$ is different from zero for some morphism $f: H \rightarrow K$ in Sub and there is a morphism $H_{0} \rightarrow H$ in Sub, then $\left(H_{0}\right)=(H)$. In the sequel we choose for any of the morphisms $f: H \rightarrow K$ in Sub a group homomorphism denoted in the same way $f: H \rightarrow K$ representing it. Recall that $f: H \rightarrow K$ is given by conjugation with an appropriate element $g \in G$. Fix $f_{0}: H_{0} \rightarrow K$ with $x_{H_{0} \cdot f_{0}} \neq 0$. We claim that the composition

$$
A: \oplus_{(H) \in I} E_{H} \circ S_{H}(L(K)) \stackrel{T(K)}{\longrightarrow} L(K) \stackrel{\operatorname{res}_{K}^{\mathrm{im}\left(f_{0}\right)}}{\longrightarrow} L\left(\mathrm{im}\left(f_{0}\right)\right) \stackrel{\mathrm{ind}_{f_{0}^{-1}} \mathrm{im}_{\left(f_{0}\right) \rightarrow H_{0}}}{\longrightarrow} L\left(H_{0}\right) \stackrel{\mathrm{pr}_{H_{0}}}{\longrightarrow} S_{H_{0}} L
$$

maps $u$ to $m \cdot x_{H_{0}, f_{0}}$ for some integer $m>0$ which is invertible in $\Lambda$. This would lead to a contradiction because of $T(K)(u)=0$ and $x_{H_{0}, f_{0}} \neq 0$.

Consider $(H) \in I$ and $\bar{f} \in J(H)$. It suffices to show that $A\left((f: H \rightarrow K) \otimes_{\Lambda\left[W_{G} H\right]} x_{H, f}\right)$ is $\left[K \cap N_{G} \operatorname{im}\left(f_{0}\right): \operatorname{im}\left(f_{0}\right)\right] \cdot x_{H, f}$ if $(H)=\left(H_{0}\right)$ and $\bar{f}=\overline{f_{0}}$, and is zero otherwise. One easily checks that $A\left((f: H \rightarrow K) \otimes_{\Lambda\left[W_{G} H\right]} x_{H, f}\right)$ is the image of $x_{H, f}$ under the composition

$$
\begin{gathered}
a(H, f): S_{H} L \stackrel{\sigma_{H}}{\longrightarrow} L(H) \stackrel{\operatorname{ind}_{f: H \rightarrow \operatorname{im}(f)}}{\longrightarrow} L(\operatorname{im}(f)) \stackrel{\operatorname{ind}_{\operatorname{im}(f)}^{K}}{\longrightarrow} L(K) \stackrel{\operatorname{res}_{K}^{\mathrm{im}\left(f_{0}\right)}}{\longrightarrow} L\left(\operatorname{im}\left(f_{0}\right)\right) \\
\stackrel{\operatorname{ind}_{f_{0}^{-1}: \operatorname{im}\left(f_{0}\right) \rightarrow H_{0}}}{\longrightarrow} L\left(H_{0}\right) \stackrel{\mathrm{pr}_{H_{0}}}{\longrightarrow} S_{H_{0}} L .
\end{gathered}
$$

The Double Coset formula implies

$$
\operatorname{res}_{K}^{\operatorname{im}\left(f_{0}\right)} \circ \operatorname{ind}_{\operatorname{im}(f)}^{K}=\sum_{k \in \operatorname{im}\left(f_{0}\right) \backslash K / \operatorname{im}(f)} \operatorname{ind}_{c(k): \operatorname{im}(f) \cap k^{-1} \operatorname{im}\left(f_{0}\right) k \rightarrow \operatorname{im}\left(f_{0}\right)} \circ \operatorname{res}_{\operatorname{im}(f)}^{\operatorname{im}(f) \cap k^{-1} \operatorname{im}\left(f_{0}\right) k} .
$$


The composition $\operatorname{pr}_{H_{0}} \circ \operatorname{ind}_{f_{0}^{-1}: \operatorname{im}\left(f_{0}\right) \rightarrow H_{0}} \circ \operatorname{ind}_{c(k): \operatorname{im}(f) \cap k^{-1} \operatorname{im}\left(f_{0}\right) k \rightarrow \operatorname{im}\left(f_{0}\right)}$ is trivial, if $c(k): \operatorname{im}(f) \cap$ $k^{-1} \operatorname{im}\left(f_{0}\right) k \rightarrow \operatorname{im}\left(f_{0}\right)$ is not an isomorphism. Suppose that $c(k): \operatorname{im}(f) \cap k^{-1} \operatorname{im}\left(f_{0}\right) k \rightarrow \operatorname{im}\left(f_{0}\right)$ is an isomorphism. Then $k^{-1} \operatorname{im}\left(f_{0}\right) k \subset \operatorname{im}(f)$. Since $H_{0}$ has been choosen maximal among the $H$ for which $x_{H, f} \neq 0$ for some morphism $f: H \rightarrow K$, this implies $x_{H, f}=0$ or that $k^{-1} \operatorname{im}\left(f_{0}\right) k=\operatorname{im}(f)$. Suppose $k^{-1} \operatorname{im}\left(f_{0}\right) k=\operatorname{im}(f)$. Then $(H)=\left(H_{0}\right)$ which implies $H=H_{0}$. Moreover, the homomorphisms in Sub represented by $f_{0}$ and $f$ agree. Hence the group homomorphisms $f_{0}$ and $f$ agree themselves and we get $k \in N_{G} \operatorname{im}\left(f_{0}\right) \cap K$. This implies that $a(H, f)=\left[K \cap N_{G} \operatorname{im}\left(f_{0}\right): \operatorname{im}\left(f_{0}\right)\right] \cdot$ id if $(H)=\left(H_{0}\right)$ and $\bar{f}=\overline{f_{0}}$, and that otherwise $a(H, f)=0$ or $x_{H, f}=0$ holds. Hence the map $T$ is injective.

(c) follows from assertion (a) and the bijectivity of the isomorphism $T$ defined in (2.3) because there is a natural isomorphism

$$
M \otimes_{\Lambda \mathrm{Sub}} E_{H} S_{H} \Lambda \otimes_{\mathbb{Z}} \operatorname{Rep}_{\mathbb{C}}(?) \stackrel{\cong}{\longrightarrow} M(H) \otimes_{\Lambda\left[W_{G} H\right]} S_{H} \Lambda \otimes_{\mathbb{Z}} \operatorname{Rep}_{\mathbb{C}}(?)
$$

Now (d) follows from (c) and the fact that the $\Lambda\left[W_{G} H\right]$-module $S_{H} \Lambda \otimes_{\mathbb{Z}} \operatorname{Rep}_{\mathbb{C}}(?) \cong \operatorname{im}\left(\theta_{C}\right)$ is projective. This finishes the proof of Theorem 3.5.

\section{The construction of the Chern character}

In this section we want to prove Theorem 0.7. There are similarities with the construction in [11]. The main difference is that here we want to give a construction, where we only have to invert the orders of elements in $\mathcal{F}(X)$, whereas in [11] we have worked over the rationals. In [11] we have used the Hurewicz homomorphism from stable homotopy to singular homology, which is only an isomorphism after inverting all primes. We will use the multiplicative structure of $K_{*}^{G}$ instead and work with a different source for the equivariant Chern character, which allows us to invert only the orders of finite subgroups of $G$.

In the sequel we denote by $K_{p}^{G}(X)$ the equivariant $K$-homology of a proper $G$ - $C W$-complex $X$. It is defined by $\operatorname{colim}_{Y \subset X} K K_{G}^{p}\left(C_{0}(Y), \mathbb{C}\right)$, where $Y$ runs over all cocompact $G$-subcomplexes of $X$ and $K K_{G}^{p}\left(C_{0}(Y), \mathbb{C}\right)$ denotes equivariant $K K$-theory of the $G$ - $C^{*}$-algebra $C_{0}(X)$ of continuous functions $X \rightarrow \mathbb{C}$, which vanish at infinity, and the $C^{*}$-algebra $\mathbb{C}$ with the trivial $G$-action. Given a homomorphism $\phi: H \rightarrow G$ of groups and a proper $H$-CW-complex, then $\operatorname{ind}_{\phi} X:=G \times_{\phi} X$ is a proper $G$-CW-complex and there is an induction homomorphism

$$
\operatorname{ind}_{\phi}: K_{0}^{H}(X) \rightarrow K_{0}^{G}\left(\operatorname{ind}_{\phi} X\right)
$$

If the kernel of $\phi$ acts freely on $X$, then $\operatorname{ind}_{\phi}$ is bijective. In particular we get for a proper $G$-CWcomplex $X$ a homomorphism

$$
K_{p}^{G}(X) \stackrel{\operatorname{ind}_{G \rightarrow\{1\}}}{\longrightarrow} K_{p}(G \backslash X)
$$

which is bijective if $G$ acts freely on $X$. There is an external product

$$
\mu: K_{p}^{G}(X) \times K_{q}^{G^{\prime}}\left(X^{\prime}\right) \rightarrow K_{p+q}^{G \times G^{\prime}}\left(X \times X^{\prime}\right)
$$

for groups $G$ and $G^{\prime}$, a proper $G-C W$-complex $X$ and a proper $G^{\prime}-C W$-complex $X^{\prime}$. External products and induction are compatible. For more information about equivariant $K$-homology and $K K$-theory we refer to [8] and in particular for the induction homomorphisms to [16].

Let $X$ be a proper $G$-CW-complex. We have introduced the $\operatorname{ring} \Lambda=\Lambda^{G}(X)$ in $(0.5)$. We want to construct for $H \in \mathcal{F}(X)$ and $p=0,1$ a $\Lambda$-homomorphism

$$
\underline{\mathrm{ch}}_{p}^{G}(X)(H): \Lambda \otimes_{\mathbb{Z}} K_{p}\left(C_{G} H \backslash X^{H}\right) \otimes_{\mathbb{Z}} \operatorname{Rep}_{\mathbb{C}}(H) \rightarrow \Lambda \otimes_{\mathbb{Z}} K_{p}^{G}(X),
$$


where $K_{p}\left(C_{G} H \backslash X^{H}\right)$ is the (non-equivariant) K-homology of the $C W$-complex $C_{G} H \backslash X^{H}$. The map will be defined by the following composition

$$
\begin{gathered}
\Lambda \otimes_{\mathbb{Z}} K_{p}\left(C_{G} H \backslash X^{H}\right) \otimes_{\mathbb{Z}} \operatorname{Rep}_{\mathbb{C}}(H) \\
\operatorname{id} \otimes_{\mathbb{Z}} K_{p}\left(\operatorname{pr}_{1} ; R\right) \otimes_{\mathbb{Z}} \text { id } \uparrow \cong \\
\Lambda \otimes_{\mathbb{Z}} K_{p}\left(E G \times_{C_{G} H} X^{H}\right) \otimes_{\mathbb{Z}} \operatorname{Rep}_{\mathbb{C}}(H) \\
\operatorname{id} \otimes_{\mathbb{Z}} \operatorname{ind}_{G_{G}} \rightarrow\{1\} \otimes j \uparrow \cong \\
\Lambda \otimes_{\mathbb{Z}} K_{p}^{C_{G} H}\left(E G \times X^{H}\right) \otimes_{\mathbb{Z}} K_{0}^{H}(*) \\
\mu \downarrow \\
\Lambda \otimes_{\mathbb{Z}} K_{p}^{C_{G} H \times H}\left(E G \times X^{H}\right) \\
\left.\operatorname{ind}_{m_{H}}\right\rfloor \cong \\
\Lambda \otimes_{\mathbb{Z}} K_{p}^{G}\left(\operatorname{ind}_{m_{H}} E G \times X^{H}\right) \\
\operatorname{id} \otimes_{\mathbb{Z}} K_{p}^{G}\left(\operatorname{ind}_{m_{H}} \operatorname{pr}_{2}\right) \downarrow \\
\Lambda \otimes_{\mathbb{Z}} K_{p}^{G}\left(\operatorname{ind}_{m_{H}} X^{H}\right) \\
\operatorname{id} \otimes_{\mathbb{Z}} K_{p}^{G}\left(v_{H}\right) \downarrow \\
K_{p}^{G}(X)
\end{gathered}
$$

Some explanations are in order. We have a left $C_{G} H$-action on $E G \times X^{H}$ by $g(e, x)=(g e, g x)$ for $g \in C_{G} H, e \in E G$ and $x \in X^{H}$. It extends to a $C_{G} H \times H$-action by letting the factor $H$ acting trivially. The map $\mathrm{pr}_{1}: E G \times_{C_{G} H} X^{H} \rightarrow C_{G} H \backslash X^{H}$ is the canonical projection. It induces an isomorphism

$$
\Lambda \otimes_{\mathbb{Z}} K_{p}\left(\operatorname{pr}_{1} ; R\right): \Lambda \otimes_{\mathbb{Z}} K_{p}\left(E G \times_{C_{G} H} X^{H}\right) \stackrel{\cong}{\longrightarrow} \Lambda \otimes_{\mathbb{Z}} K_{p}\left(C_{G} H \backslash X^{H}\right)
$$

since each isotropy group of the $C_{G} H$-space $X^{H}$ is finite and for any finite group $L$ the projection induces an isomorphism $\Lambda \otimes_{\mathbb{Z}} H_{p}(B L) \stackrel{\cong}{\longrightarrow} \Lambda \otimes_{\mathbb{Z}} H_{p}(*)$ and hence by the Atiyah-Hirzebruch spectral sequence an isomorphism $\Lambda \otimes_{\mathbb{Z}} K_{p}(B L) \stackrel{\cong}{=} \Lambda \otimes_{\mathbb{Z}} K_{p}(*)$ for all $p$. The isomorphism $j: K_{0}^{H}(*) \cong$ $\operatorname{Rep}_{\mathbb{C}}(H)$ is the canonical isomorphism. The group homomorphism $m_{H}: C_{G} H \times H \rightarrow G$ sends $(g, h)$ to $g h$. We denote by $\operatorname{pr}_{2}: E G \times X^{H} \rightarrow X^{H}$ the canonical projection. The $G$-map $v_{H}: \operatorname{ind}_{m_{H}} X^{H}=$ $G \times{ }_{m_{H}} X^{H} \rightarrow X$ sends $(g, x)$ to $g x$.

Notice that we obtain a contravariant Sub-module $K_{0}\left(C_{G} ? \backslash X^{?}\right)$ by assigning to a finite subgroup $H \subset G$ the $\Lambda$-module $\Lambda \otimes_{\mathbb{Z}} K_{p}\left(C_{G} H \backslash X^{H}\right)$. We have already introduced the covariant $\Lambda$-module $\Lambda \otimes_{\mathbb{Z}} \operatorname{Rep} \mathbb{C}_{\mathbb{C}}(?)$. Analogously to [11] one checks that the various maps $\underline{\mathrm{ch}}_{p}^{G}(X)(H)$ defined above induce a map of $\Lambda$-modules

$$
\operatorname{ch}_{p}^{G}(X): \Lambda \otimes_{\mathbb{Z}} K_{p}\left(C_{G} ? \backslash X^{?}\right) \otimes_{\Lambda \text { Sub }} \Lambda \otimes_{\mathbb{Z}} \operatorname{Rep}_{\mathbb{C}}(?) \rightarrow \Lambda \otimes_{\mathbb{Z}} K_{p}^{G}(X) .
$$

Notice that for $L \in \mathcal{F}(X)$ and $X=G / L$ the $\Lambda$ Sub-module $K_{0}\left(C_{G} ? \backslash(G / L)^{?}\right)$ is isomorphic to the $\Lambda$ Sub-module $\Lambda$ morsub $(?, L)$, which sends a finite subgroup $H \subset G$ to the free $\Lambda$-module with base $\operatorname{mor}_{\mathrm{sub}}(H, K)$. By the Yoneda Lemma one obtaines a canonical isomorphism

$$
\Lambda \otimes_{\mathbb{Z}} K_{p}\left(C_{G} ? \backslash(G / L)^{?}\right) \otimes_{\Lambda \text { Sub }} \Lambda \otimes_{\mathbb{Z}} \operatorname{Rep}_{\mathbb{C}}(?) \stackrel{\cong}{\longrightarrow} \operatorname{Rep}_{\mathbb{C}}(L) .
$$

One easily checks that under this identification $\operatorname{ch}_{0}^{G}(G / L)$ becomes the canonical identification of $\operatorname{Rep}_{\mathbb{C}}(L)$ with $K_{0}^{G}(G / L)$. Notice that $K_{1}\left(C_{G} ? \backslash(G / L)^{?}\right)$ and $K_{1}^{G}(G / L)$ are both trivial. Hence 
$\operatorname{ch}_{p}^{G}(G / L)$ is bijective for all $L \in \mathcal{F}(X)$ and $p=0,1$. Because of Theorem 3.5 (d) the source of $\mathrm{ch}_{*}^{G}$ is an equivariant homology theory on proper $G$-CW-complexes $Y$ with $\mathcal{F}(Y) \subset \mathcal{F}(X)$. One easily checks that $\mathrm{ch}_{*}^{G}$ is compatible with the Mayer-Vietoris sequences. By induction over the number of equivariant cells and the Five-Lemma $\operatorname{ch}_{p}^{G}(Y)$ is bijective for any finite proper $G$-CW-complex $Y$ with $\mathcal{F}(Y) \subset \mathcal{F}(X)$. Notice that $K_{p}^{G}(Y)$ is the colimit $\operatorname{colim}_{Z \subset Y} K_{p}^{G}(Z)$, where $Z$ runs through all finite $G$-CW-subcomplexes $Z$ of $Y$. The analogous statement holds for the source of $\operatorname{ch}_{*}^{G}$. Hence $\operatorname{ch}_{p}^{G}(Y)$ is bijective for all proper $G$-CW-complexes $Y$ with $\mathcal{F}(Y) \subset \mathcal{F}(X)$ and $p=0,1$. Now Theorem 0.7 follows from Theorem 3.5 (c).

\section{The Baum-Connes Conjecture and the Trace Conjecture}

In the sequel we denote for a proper $G$-CW-complex $X$ by

$$
\operatorname{asmb}^{G}: K_{0}^{G}(X) \rightarrow K_{0}\left(C_{r}^{*}(G)\right)
$$

the assembly map which essentially assigns to an element in $K_{0}^{G}(X)$ represented by an equivariant Kasparov cycle its index. Given a homomorphism $\phi: H \rightarrow G$ of groups with finite kernel, there is an induction homomorphism $\operatorname{ind}_{\phi}: K_{p}\left(C_{r}^{*}(H)\right) \rightarrow K_{p}\left(C_{r}^{*}(G)\right)$ such that the following diagram commutes $[16$, Theorem 1]

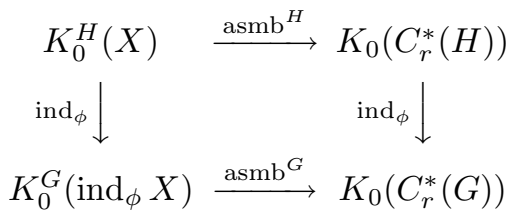

These induction homomorphisms, the assembly maps and the change of rings homomorphisms associated to the passage from $C_{r}^{*}(G)$ to $\mathcal{N}(G)$ are compatible with the external products

$$
\begin{aligned}
\mu: K_{p}^{G}(X) \times K_{q}^{G^{\prime}}\left(X^{\prime}\right) & \rightarrow K_{p+q}^{G \times G^{\prime}}\left(X \times X^{\prime}\right) ; \\
\mu: K_{p}\left(C_{r}^{*}(G)\right) \times K_{q}\left(C_{r}^{*}\left(G^{\prime}\right)\right) & \rightarrow K_{p+q}\left(C_{r}^{*}\left(G \times G^{\prime}\right)\right) ; \\
\mu: K_{p}(\mathcal{N}(G)) \times K_{q}\left(\mathcal{N}\left(G^{\prime}\right)\right) & \rightarrow K_{p+q}\left(\mathcal{N}\left(G \times G^{\prime}\right)\right)
\end{aligned}
$$

for groups $G$ and $G^{\prime}$, a proper $G$-CW-complex $X$ and a proper $G^{\prime}-C W$-complex $X^{\prime}$. We will use in the sequel the elementary fact that for any $G$-map $f: X \rightarrow Y$ of proper $G$ - $C W$-complexes the composition $K_{0}^{G}(X) \stackrel{K_{0}^{G}(f)}{\longrightarrow} K_{0}^{G}(Y) \stackrel{\operatorname{asmb}^{G}}{\longrightarrow} K_{0}\left(C_{r}^{*}(G)\right)$ is $\operatorname{asmb}^{G}: K_{0}^{G}(X) \rightarrow K_{0}\left(C_{r}^{*}(G)\right)$. In the sequel the letter $i$ denotes change of rings homomorphism for the canonical map $C_{r}^{*}(G) \rightarrow \mathcal{N}(G)$.

Let $X$ be a proper $G$-CW-complex. We have introduced $J=J^{G}(X)$ in (0.6). Define the homomorphism

$$
\begin{aligned}
\xi_{1}: \oplus_{(C) \in J} \Lambda \otimes_{\mathbb{Z}} K_{0}\left(C_{G} C \backslash X^{C}\right) \otimes_{\Lambda\left[W_{G} C\right]} \operatorname{im}\left(\theta_{C}: \Lambda \otimes_{\mathbb{Z}} \operatorname{Rep}_{\mathbb{C}}(C) \rightarrow \Lambda \otimes_{\mathbb{Z}} \operatorname{Rep}_{\mathbb{C}}(C)\right) & \rightarrow K_{0}(\mathcal{N}(G))
\end{aligned}
$$

by the composition of the equivariant Chern character of Theorem 0.7

$$
\begin{aligned}
\operatorname{ch}_{0}^{G}(X): \oplus_{(C) \in J} \Lambda \otimes_{\mathbb{Z}} K_{0}\left(C_{G} C \backslash X^{C}\right) \otimes_{\Lambda\left[W_{G} C\right]} \operatorname{im}\left(\theta_{C}: \Lambda \otimes_{\mathbb{Z}} \operatorname{Rep}_{\mathbb{C}}(C) \rightarrow \Lambda \otimes_{\mathbb{Z}} \operatorname{Rep}_{\mathbb{C}}(C)\right) & \stackrel{\cong}{\longrightarrow} \Lambda \otimes_{\mathbb{Z}} K_{0}^{G}(X),
\end{aligned}
$$

the assembly map

$$
\mathrm{id} \otimes \operatorname{asmb}^{G}: \Lambda \otimes_{\mathbb{Z}} K_{0}^{G}(X) \rightarrow \Lambda \otimes_{\mathbb{Z}} K_{0}\left(C_{r}^{*}(G)\right)
$$


and the change of rings homomorphism

$$
\operatorname{id} \otimes i: \Lambda \otimes_{\mathbb{Z}} K_{0}\left(C_{r}^{*}(G)\right) \rightarrow \Lambda \otimes_{\mathbb{Z}} K_{0}(\mathcal{N}(G)) .
$$

This is the homomorphism which we want to understand. In particular we are interested in its image. We will identify it with a second easier to compute homomorphism

$$
\begin{aligned}
\xi_{2}: \oplus_{(C) \in J} \Lambda \otimes_{\mathbb{Z}} K_{0}\left(C_{G} C \backslash X^{C}\right) \otimes_{\Lambda\left[W_{G} C\right]} \operatorname{im}\left(\theta_{C}: \Lambda \otimes_{\mathbb{Z}} \operatorname{Rep}_{\mathbb{C}}(C) \rightarrow \Lambda \otimes_{\mathbb{Z}} \operatorname{Rep}_{\mathbb{C}}(C)\right) & \rightarrow K_{0}(\mathcal{N}(G)),(5
\end{aligned}
$$

which is defined as follows. Let $l: \operatorname{im}\left(\theta_{C}\right) \rightarrow \operatorname{Rep}_{\mathbb{C}}(C)$ be the inclusion. Let $K_{0}(\mathrm{pr}): K_{0}\left(C_{G} C \backslash X^{C}\right) \rightarrow$ $K_{0}(*)$ be induced by the projection from $C_{G} C \backslash X^{C}$ to the one-point space *. We obtain a map

$$
\left(i \circ \operatorname{asmb}^{\{1\}} \circ K_{0}(\mathrm{pr})\right) \otimes l: K_{0}\left(C_{G} C \backslash X^{C}\right) \otimes \operatorname{im}\left(\theta_{C}\right) \rightarrow K_{0}(\mathcal{N}(\{1\})) \otimes \operatorname{Rep}_{\mathbb{C}}(C) .
$$

Define

$$
\alpha: K_{0}(\mathcal{N}(\{1\})) \otimes \operatorname{Rep}_{\mathbb{C}}(C) \rightarrow \operatorname{Rep}_{\mathbb{C}}(C) \quad[U] \otimes[W] \mapsto \operatorname{dim}_{\mathbb{C}}(U) \cdot[W] .
$$

Notice that $\alpha$ is essentially given by the external product and $K_{0}(\mathcal{N}(H))=\operatorname{Rep}_{\mathbb{C}}(H)$ holds by definition for any finite group $H$. Induction yields a map

$$
\operatorname{ind}_{C}^{G}: K_{0}(\mathcal{N}(C)) \rightarrow K_{0}(\mathcal{N}(G)) .
$$

The composition of these three maps above induces for any finite cyclic subgroup $C \subset G$ a homomorphism

$$
\xi_{2}(C): \Lambda \otimes_{\mathbb{Z}} K_{0}\left(C_{G} C \backslash X^{C}\right) \otimes_{\Lambda\left[W_{G} C\right]} \operatorname{im}\left(\theta_{C}: \Lambda \otimes_{\mathbb{Z}} \operatorname{Rep}_{\mathbb{C}}(C) \rightarrow \Lambda \otimes_{\mathbb{Z}} \operatorname{Rep}_{\mathbb{C}}(C)\right) \rightarrow K_{0}(\mathcal{N}(G)) .
$$

Define $\xi_{2}$ to be the direct sum $\oplus_{(C) \in J} \xi_{2}(C)$ after the choice of a representative $C \in(C)$ for each $(C) \in J$.

Theorem 5.4 Let $X$ be a proper $G-C W$-complex. Then the maps $\xi_{1}$ of (5.2) and $\xi_{2}$ of (5.3) agree.

Proof : In the sequel maps denoted by the letter $\mu$ will be given by external products and pr denotes the projection from a space to the one-point space $*$. Fix a cyclic subgroup $C \in \mathcal{F}(X)$. Notice that the homomorphism $m_{C}: C_{G} C \times C \rightarrow G \quad(g, c) \mapsto g c$ has a finite kernel so that induction is defined also on the level of the reduced group $C^{*}$-algebra and the group von Neumann algebra. Denote by $\nu: \Lambda \otimes_{\mathbb{Z}} K_{0}^{C_{G} C \times C}\left(E G \times X^{C}\right) \rightarrow K_{0}^{G}(X)$ the composition of the maps id $\otimes K_{0}^{G}\left(v_{C}\right)$, id $\otimes K_{0}^{G}\left(\operatorname{ind}_{m_{C}} \operatorname{pr}_{2}\right)$ and ind $m_{C}$ appearing in the definition of $\underline{\mathrm{ch}}_{0}(X)(C)$. Then the following diagram commutes

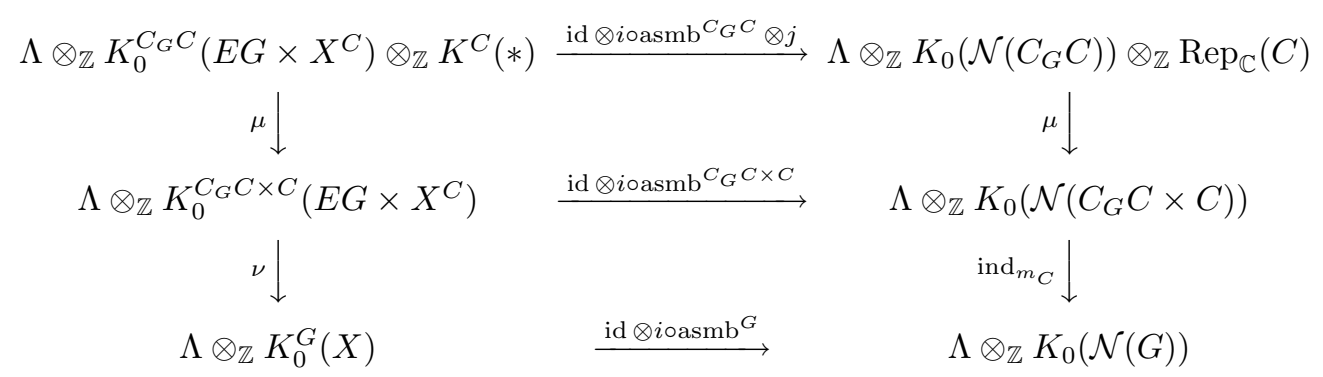

For any group $G$ the map induced by the center-valued von Neumann dimension

$$
\operatorname{dim}_{\mathcal{N}(G)}^{u}: K_{0}(\mathcal{N}(G)) \quad \rightarrow \quad \mathcal{Z}(\mathcal{N}(G))
$$


is injective. Given a $C W$-complex $Z$ and an element $\eta \in K_{0}(Z)$, there is a closed manifold $M$ with a map $f: M \rightarrow B G$ and an elliptic complex $D^{*}$ of differential operators of order 1 over $M$ such that $K_{0}(f): K_{0}(M) \rightarrow K_{0}(Z)$ maps the class $\left[D^{*}\right] \in K_{0}(M)$ to $\eta[2]$. In the case $Z=B G$ the composition

$$
K_{0}(M) \stackrel{K_{0}(f)}{\longrightarrow} K_{0}(B G) \stackrel{\left(\operatorname{ind}_{G \rightarrow\{1\}}\right)^{-1}}{\longrightarrow} K_{0}^{G}(E G) \stackrel{\operatorname{asmb}^{G}}{\longrightarrow} K_{0}\left(C_{r}^{*}(G)\right) \stackrel{i}{\rightarrow} K_{0}(\mathcal{N}(G)) \stackrel{\operatorname{dim}_{\mathcal{N}(G)}^{u}}{\longrightarrow} \mathcal{Z}(\mathcal{N}(G))
$$

resp. the composition

$$
K_{0}(M) \stackrel{K_{0}(\mathrm{pr})}{\longrightarrow} K_{0}(*) \stackrel{\operatorname{asmb}^{\{1\}}}{\longrightarrow} K_{0}\left(C^{*}(\{1\}) \stackrel{i}{\longrightarrow} K_{0}(\mathcal{N}(\{1\})) \stackrel{\operatorname{ind}_{\{1\}}^{G}}{\longrightarrow} K_{0}(\mathcal{N}(G)) \stackrel{\operatorname{dim}_{\mathcal{N}(G)}^{u}}{\longrightarrow} \mathcal{Z}(\mathcal{N}(G))\right.
$$

maps $\left[D^{*}\right]$ to the element $\operatorname{index}_{\mathcal{N}(G)}^{u}\left(\bar{D}^{*}\right) \operatorname{resp}$. index $\left(D^{*}\right) \cdot 1_{\mathcal{N}(G)}$, where $\operatorname{index}_{\mathcal{N}(G)}^{u}\left(\bar{D}^{*}\right) \operatorname{resp}$. index $\left(D^{*}\right)$ has been defined in (1.7) resp. (1.1). We conclude from Theorem 0.4 and the injectivity of the map $\operatorname{dim}_{\mathcal{N}(G)}^{u}$ of (5.5) that the following diagram commutes

$$
\begin{array}{rll}
K_{0}^{G}(E G) & \stackrel{i \circ \mathrm{asmb}^{G}}{\longrightarrow} & K_{0}(\mathcal{N}(G)) \\
K_{0}(\mathrm{pr}) \mathrm{oind}_{G \rightarrow\{1\}}^{-1} \downarrow & \operatorname{ind}_{\{1\}}^{G} \uparrow \\
K_{0}(*) & \stackrel{i \circ \mathrm{asmb}^{\{1\}}}{\longrightarrow} & K_{0}(\mathcal{N}(\{1\}))
\end{array}
$$

Since there is a $C_{G} C$-map $E G \times X^{C} \rightarrow E C_{G} C$, we conclude from the diagram above applied to the case $G=C_{G} C$ that the following diagramm commutes

$$
\begin{array}{rr}
\Lambda \otimes_{\mathbb{Z}} K_{0}^{C_{G} C}\left(E G \times X^{C}\right) \otimes_{\mathbb{Z}} K^{C}(*) \stackrel{\text { id } \otimes i o a s m b^{C_{G} C} \otimes j}{\longrightarrow} & \Lambda \otimes_{\mathbb{Z}} K_{0}\left(\mathcal{N}\left(C_{G} C\right)\right) \otimes_{\mathbb{Z}} \operatorname{Rep}_{\mathbb{C}}(C) \\
\operatorname{id} \otimes\left(K_{0}(\operatorname{pr}) \operatorname{oind}_{C_{G} C \rightarrow\{1\}}\right) \otimes j \downarrow & \uparrow \text { id } \otimes \operatorname{ind}_{\{1\}}^{C_{G} C} \otimes \text { id } \\
\Lambda \otimes_{\mathbb{Z}} K_{0}(*) \otimes_{\mathbb{Z}} \operatorname{Rep}_{\mathbb{C}}(C) \quad \stackrel{\mathrm{id} \otimes i o \operatorname{asmb}^{\{1\}} \otimes \mathrm{id}}{\longrightarrow} \Lambda \otimes_{\mathbb{Z}} K_{0}\left(\mathcal{N}(\{1\}) \otimes_{\mathbb{Z}} \operatorname{Rep}_{\mathbb{C}}(C)\right.
\end{array}
$$

The composition

$$
K_{0}\left(\mathcal{N}(\{1\}) \otimes_{\mathbb{Z}} \operatorname{Rep}_{\mathbb{C}}(C) \stackrel{\operatorname{ind}_{\{1\}}^{C_{G} C} \otimes \mathrm{id}}{\longrightarrow} K_{0}\left(\mathcal{N}\left(C_{G} C\right)\right) \otimes_{\mathbb{Z}} \operatorname{Rep}_{\mathbb{C}}(C) \stackrel{\mu}{\longrightarrow} K_{0}\left(\mathcal{N}\left(C_{G} C \times C\right)\right) \stackrel{\operatorname{ind}_{m_{C}}}{\longrightarrow} K_{0}(\mathcal{N}(G))\right.
$$

agrees with the composition

$$
K_{0}(\mathcal{N}(\{1\})) \otimes \operatorname{Rep}_{\mathbb{C}}(C) \stackrel{\alpha}{\longrightarrow} \operatorname{Rep}_{\mathbb{C}}(C)=K_{0}(\mathcal{N}(C)) \stackrel{\operatorname{ind}_{C}^{G}}{\longrightarrow} K_{0}(\mathcal{N}(G)) .
$$

We conclude that the following diagram commutes for any cyclic subgroup $C \in \mathcal{F}(X)$

$$
\begin{aligned}
& \Lambda \otimes_{\mathbb{Z}} K_{0}^{C_{G} C}\left(E G \times X^{C}\right) \otimes_{\mathbb{Z}} K^{C}(*) \stackrel{\left(\text { id } \otimes i \circ a_{a s m b} C_{G}^{C}\right) \circ \nu \circ \mu}{\longrightarrow} \Lambda \otimes_{\mathbb{Z}} K_{0}(\mathcal{N}(G)) \\
& \text { id } \otimes\left(i \circ \operatorname{asmb}^{\{1\}} \circ K_{0}(\text { pr }) \operatorname{oind}_{C_{G} C \rightarrow\{1\}}\right) \otimes j \downarrow \\
& \Lambda \otimes_{\mathbb{Z}} K_{0}(\mathcal{N}(\{1\})) \otimes_{\mathbb{Z}} \operatorname{Rep}_{\mathbb{C}}(C) \quad \stackrel{\operatorname{id} \otimes \alpha}{\longrightarrow} \quad \Lambda \otimes_{\mathbb{Z}} \operatorname{Rep}_{\mathbb{C}}(C)
\end{aligned}
$$

Hence the following diagram commutes for any cyclic subgroup $C \in \mathcal{F}(X)$

$$
\begin{array}{ccl}
\Lambda \otimes_{\mathbb{Z}} K_{p}\left(C_{G} C \backslash X^{C}\right) \otimes_{\mathbb{Z}} \operatorname{Rep}_{\mathbb{C}}(C) \stackrel{\text { id } \otimes\left(\alpha \circ\left(i \circ \mathrm{asmb}^{\{1\}} \circ K_{0}(\mathrm{pr}) \otimes \mathrm{id}\right)\right.}{\longrightarrow} & \Lambda \otimes_{\mathbb{Z}} \operatorname{Rep}_{\mathbb{C}}(C) \\
\operatorname{id~} \otimes \underline{\mathrm{ch}}_{0}^{G}(X)(C) \downarrow & \operatorname{id~} \otimes \operatorname{ind}_{C}^{G} \downarrow \\
\Lambda \otimes_{\mathbb{Z}} K_{0}^{G}(X) & \stackrel{\text { id } \otimes\left(i \circ \mathrm{asmb}^{G}\right)}{\longrightarrow} & \Lambda \otimes_{\mathbb{Z}} K_{0}(\mathcal{N}(G))
\end{array}
$$

Now Theorem 5.4 (and hence also Theorem 0.8) follow. 


\section{References}

[1] Atiyah, M.: "Elliptic operators, discrete groups and von Neumann algebras", Astérisque 32, 43-72 (1976).

[2] Baum, P. and Douglas, R.G.: "K-homology and index theory", in "Operator algebras and applications", editor: Kadison, R.V., Proc. Symp. Pure Math. 38 (Part 1), AMS, Providence, $117-173$ (1982).

[3] Baum, P. and Connes, A.: "Geometric K-theory for Lie groups and foliations", BrownUniversity/IHES-preprint 1982, appeared in L'Enseignement Mathématique 46, 3-42 (2000).

[4] Baum, P. and Connes, A.: "Chern character for discrete groups", in: Matsumoto, Miyutami, and Morita (eds.): "A fête of topology; dedicated to Tamura", 163-232, Academic Press (1988).

[5] Baum, P., Connes, A., and Higson, N.: "Classifying space for proper actions and $K$-theory of group $C^{*}$-algebras", in: Doran, R.S. (ed.): " $C^{*}$-algebras", Contemporary Mathematics 167, 241-291 (1994).

[6] Cheeger, J., Gromov, M., and Taylor, M.: "Finite propagation speed, kernel estimates for functions of the Laplace operator, and the geometry of complete Riemannian manifolds", Journal of Differential Geometry 17, 15-53 (1982).

[7] Kadison, R.V. and Ringrose, J.R.: "Fundamentals of the theory of operator algebras, volume II: Advanced theory", Pure and Applied Mathematics, Academic Press (1986).

[8] Kasparov, G.G.: "Equivariant KK-theory and the Novikov conjecture", Inventiones Mathematicae 91, 147-201 (1988).

[9] Lott, J.: "Heat kernels on covering spaces and topological invariants", Journal of Differential Geometry 35, 471-510 (1992).

[10] Lück, W., "Transformation groups and algebraic K-theory", Lecture Notes in Mathematics 1408 (1989).

[11] Lück, W., "Chern characters for proper equivariant homology theories and applications to Kand L-theory", Preprintreihe SFB 478 - Geometrische Strukture in der Mathematik, Heft 104, Münster (2000).

[12] Mishchenko, A.S. and Fomenko, A.T.: "The index of elliptic operators over $C^{*}$-algebras", Math. USSR Izv. 15, 87-112 (1980).

[13] Roy, R.: "The trace conjecture - a counterexample", K-theory 17, 209-213 (1999).

[14] Roy, R.: "A counterexample to questions on the integrality property of virtual signature", Topology and its applications 100, 177-185 (2000).

[15] Serre, J.-P.: "Linear representations of finite groups", Springer-Verlag (1977).

[16] Valette, A.: "On the Baum-Connes assembly map", appendix to "Introduction to the BC conjecture", to appear as ETHZ lecture notes, Birkhauser. 